\title{
Lepton flavour violation: physics potential of a Linear Collider
}

\author{
A. Abada, ${ }^{a}$ A.J.R. Figueiredo, ${ }^{b, c}$ J.C. Romão ${ }^{b}$ and A.M. Teixeira ${ }^{c}$ \\ ${ }^{a}$ Laboratoire de Physique Théorique, CNRS-UMR 8627, Université de Paris-Sud 11, \\ F-91405 Orsay Cedex, France \\ ${ }^{b}$ Centro de Física Teórica de Partículas, Instituto Superior Técnico, \\ Av. Rovisco Pais 1, 1049-001 Lisboa, Portugal \\ ${ }^{c}$ Laboratoire de Physique Corpusculaire, CNRS/IN2P3-UMR 6533, \\ Campus des Cézeaux, 24 Av. des Landais, F-6317y Aubière Cedex, France \\ E-mail: abada@th.u-psud.fr, ajrf@cftp.ist.utl.pt, \\ jorge.romao@ist.utl.pt, ana.teixeira@clermont.in2p3.fr
}

ABSTRACT: We revisit the potential of a Linear Collider concerning the study of lepton flavour violation, in view of new LHC bounds and of the (very) recent developments in lepton physics. Working in the framework of a type I supersymmetric seesaw, we evaluate the prospects of observing seesaw-induced lepton flavour violating final states of the type $e \mu$ plus missing energy, arising from $e^{+} e^{-}$and $e^{-} e^{-}$collisions. In both cases we address the potential background from standard model and supersymmetric charged currents. We also explore the possibility of electron and positron beam polarisation. The statistical significance of the signal, even in the absence of kinematical and/or detector cuts, renders the observation of such flavour violating events feasible over large regions of the parameter space. We further consider the $\mu^{-} \mu^{-}+E_{\text {miss }}^{T}$ final state in the $e^{-} e^{-}$beam option finding that, due to a very suppressed background, this process turns out to be a truly clear probe of a supersymmetric seesaw, assuming the latter to be the unique source of lepton flavour violation.

KEYwords: Supersymmetry Phenomenology

ARXIV EPRINT: 1206.2306 


\section{Contents}

1 Introduction 1

2 LFV in the SUSY seesaw $\quad 4$

3 LFV at a Linear Collider: $e^{+} e^{-}$beam option 6

4 LFV at a Linear Collider: $e^{-} e^{-}$beam option 9

5 LC potential for LFV discovery $\quad 10$

$\begin{array}{lll}5.1 & e^{+} e^{-} \text {beam option } & 14\end{array}$

$\begin{array}{ll}5.2 e^{-} e^{-} \text {beam option } & 19\end{array}$

5.3 cLFV from $\mu^{-} \mu^{-}$final states 22

6 Concluding remarks $\quad 24$

$\begin{array}{ll}\text { A Particle production and decay } & 26\end{array}$

\section{Introduction}

Although the Large Hadron Collider (LHC) is yet to discover supersymmetry (SUSY), this class of models remains one of the most appealing extensions of the Standard Model (SM). A very attractive explanation for the smallness of neutrino masses, $m_{\nu}$, and their mixings [1] is then to embed a seesaw mechanism in the framework of SUSY models. A high scale (close to the grand unification — GUT — scale) type I seesaw [2-6] can account for $m_{\nu}$ and the observed pattern of the mixing angles, for naturally large values of the Yukawa couplings, $Y^{\nu}$. Such scenarios are also very appealing as they can explain the observed baryon asymmetry of the Universe (BAU) via leptogenesis [7] (through the CP and lepton number violating decays of the heavy mediators responsible for the smallness of neutrino masses, i.e., the right-handed neutrinos). Furthermore, when realised at such high scales, the new dynamics has only a marginal effect on electroweak (EW) precision measurements and observables [8], contrarily to other seesaw realisations as, for instance, in the case of a type II seesaw [9-15]. By itself, and independently of the scale at which it is realised, the type I seesaw is very hard to probe - directly or indirectly - as the mediators are very difficult to produce at colliders (in particular when heavier than the TeV scale) and/or their effects at low-energies are extremely small (typically in association with small couplings). However, when embedded in the framework of SUSY models - the so-called SUSY seesaw - the type I seesaw offers the interesting feature that flavour violation in the slepton sector is radiatively induced [16] and, at low-energies, the new contributions to a 
large array of charged lepton flavour violation (cLFV) observables might provide a unique probe of this mass generation mechanism.

In the framework of a type I SUSY seesaw, low-energy cLFV observables such as $\ell_{i} \rightarrow \ell_{j} \gamma, \ell_{i} \rightarrow 3 \ell_{j}, \mu-e$ conversion in nuclei, etc., have been extensively addressed [1739]. Moreover, cLFV has also been studied in high-energy observables [40-52], as those that can be probed at the LHC as, for instance, lepton flavour violating neutralino decays and slepton mass splittings. The impact of a type I seesaw on the SUSY Higgs spectrum has been recently discussed in [53]. Furthermore, under the assumption of a unique source of Lepton Flavour Violation (LFV), one can rely on the synergy of high- and low-energy observables and devise powerful probes (using data from neutrino experiments, rare lepton decays, and collider searches), which allow to shed light on the fundamental nature of LFV.

At hadron colliders (such as the Tevatron and the LHC) sleptons are in general only produced in the decay chains of a strongly interacting sparticle, i.e. squarks or gluinos; if these states are heavy (as suggested by the negative searches at the LHC), then slepton production, even if the latter are comparatively light, is extremely difficult (occurring only via EW gaugino production, which is typically subdominant). Nevertheless, numerous slepton-dedicated studies have been conducted for the LHC, in particular focusing on the case in which sleptons are produced from gaugino decays. Hence, the studies of cLFV observables are necessarily associated with specific decay chains that only occur in (reduced) regions of the SUSY parameter space: in general, cLFV is studied through dilepton mass distributions $\left(m_{\ell \ell}\right)$ of $\chi_{2}^{0} \rightarrow \ell_{i} \ell_{j} \chi_{1}^{0}$, via observables such as additional edges in $m_{\ell \ell}$ and slepton mass splittings (see, for example [42, 44-50]).

A high-energy lepton collider, such as ILC [54-56], CLIC [57] or a muon collider [58], will allow to extend and to complement LHC searches. Following a potential discovery of new physics at the LHC, linear colliders offer numerous advantages for precision studies, in particular the possibility of probing the properties of the new states. Linear lepton Colliders (LC) constitute an excellent laboratory to study the slepton sector (see, e.g. [59-63]). In contrast to hadron colliders, the exact nature of the colliding particles is known in a Linear Collider - valid for electron and muon colliders - and the polarisation of the beams can be known with high precision. In fact, the possibility of beam polarisation is instrumental to either suppress or enhance SM contributions, so that the latter can be differentiated from new physics processes. It has been extensively pointed out that the ability of adjusting the polarisation of each beam independently (and simultaneously) opens unique possibilities to test the properties of the produced particles: their quantum numbers and "chiral" couplings can thus be directly probed under a minimal set of assumptions [64, 65].

In the case of supersymmetric models, a LC will complete the LHC exploration of SUSY [66], possibly resolving particularly difficult cases where LHC measurements fail to clearly identify SUSY particles, and determining the properties of the new particles (for instance, CP properties [67-69], the Majorana nature of neutralinos [70, 71] and of other new states [72], etc.). Concerning the slepton sector, and despite a possibly heavy squark and gluino sectors, lighter sleptons can be directly produced at a Linear Collider, operating at a comparatively smaller centre of mass (c.o.m.) energy, $\sqrt{s}$. In addition to very precise measurements of slepton parameters, such a clean environment (with a low 
hadronic background when compared to the LHC) further allows dedicated studies of rare processes, as is the case of flavour violation.

SUSY cLFV processes at a Linear Collider, such as for example $e^{ \pm} e^{-} \rightarrow \ell_{i}^{ \pm} \ell_{j}^{-}+$ $E_{\text {miss }}^{T}(+$ jets $)$ and $e^{ \pm} e^{-} \rightarrow \ell_{i}^{ \pm} \ell_{j}^{-}$, have been previously addressed in the literature. Early analyses have treated flavour violation in the slepton sector using a sneutrino mixing (oscillation) based approach [73-76], while recent studies considered an effective parametrization of the amount of LFV [77], in both cases without focusing on a specific mechanism of flavour violation in the lepton sector. Other studies have specifically considered type I seesaw-inspired models $[78,79]$. A Linear Collider could also offer the possibility of working in the so called $e \gamma$ or $\gamma \gamma$ modes - thus becoming a very high energy photon collider, with the option of polarised photon beams [80,81]. cLFV has also been considered in $\gamma \gamma \rightarrow \ell_{i} \ell_{j}$ collisions using a phenomenological effective approach [82].

In recent years, not only neutrino parameters have been better constrained [83], but crucial data has just been obtained by several collaborations, with very precise measurements of the Chooz angle [84-88]. Furthermore, negative LHC SUSY searches have also suggested that the SUSY spectrum might be far heavier than what had previously been considered [89-107]. Moreover, the sensitivity of the experiments dedicated to the observation of low-energy cLFV transitions has also significantly improved [108], especially in what concerns $\mu \rightarrow e \gamma$ decays [109] and $\mu-e$ conversion in nuclei [110, 111].

Parallel to these achievements, recent technological developments potentially suggest that the future Linear Collider will be an ambitious project concerning the expected detector efficiency, as well as electron and positron beam polarisations. It is also expected that such a collider will run at (very) large centre of mass energies, leading to a very high luminosity - see e.g. $[56,57]$. In view of all the above, in this study we revisit the potential manifestations of cLFV in a future LC assuming a type I SUSY seesaw as a unique source of LFV, discussing how the possible direct signals and their synergy with other strongly correlated cLFV observables can contribute to probe the mechanism of neutrino mass generation.

In this work we study cLFV in association to slepton and gaugino pair production in both $e^{+} e^{-}$and $e^{-} e^{-}$collisions. In view of the (recent) stringent experimental bounds on flavour violation in the $\mu-e$ sector, we only consider final states involving muons and/or electrons, further allowing to maximally benefit from the efficiency of the muon detectors. Let us recall that the reconstruction of high-energy electrons and muons is a key feature of a LC - for instance in the case of CLIC [57, 59], the muon and electron average identification efficiencies are expected to be as high as $99 \%$ and $96 \%$, respectively. However, we do allow for (intermediate) tau production, focusing exclusively on its purely leptonic decays, $\tau \rightarrow \nu_{\tau} \ell \bar{\nu}_{\ell}$. We thus consider all processes leading to final states involving two charged leptons $(\mu, e)$ and missing energy. In the presence of flavour violation in the neutrino sector, as confirmed by neutrino data, potential LFV backgrounds arise from charged lepton currents: even in the absence of a seesaw mechanism, these backgrounds are present both in the SM as well as in its Minimal Supersymmetric extension (MSSM), once neutrino oscillations are accommodated via the leptonic mixing matrix, $U_{\text {MNS }}[112,113]$. Thus, in addition to the pure SUSY seesaw signal, both the SM and SUSY charged current 
interactions provide important contributions to $e^{ \pm} e^{-} \rightarrow \ell_{i}^{ \pm} \ell_{j}^{-}+E_{\text {miss }}^{T}(i \neq j)$. While in the "signal" $E_{\text {miss }}^{T}$ is strictly composed of SUSY neutral particles escaping the detector (the lightest SUSY particle (LSP), which is the lightest neutralino, $\chi_{1}^{0}$ ), in the SM (MSSM) $E_{\text {miss }}^{T}$ is in the form of neutrinos (neutrinos + neutralinos).

In addition to different flavour final states, we also discuss the unique potential of the $e^{-} e^{-} \rightarrow \mu^{-} \mu^{-}+E_{\text {miss }}^{T}$ channel, since in this case the background is considerably suppressed - in this case the SM contributions to the background are essentially non-existent. In view of such a clean signal, this process deserves a careful analysis since, in addition to directly probing the Majorana nature of the fermionic neutral superparticles, it also allows to relate the neutrino mass generation mechanism to a cLFV high-energy observable.

The purpose of the present study is to emphasise the potential of a future Linear Collider in disentangling a SUSY seesaw induced Lepton Flavour Violation from the potential SM and SUSY backgrounds. We assume centre of mass energies between $500 \mathrm{GeV}$ and $3 \mathrm{TeV}$ and integrated luminosities of 0.5 and $3 \mathrm{ab}^{-1}$, and we explore the possibility of electron and positron beam polarisation, comparing the significance of the signal for different beam configurations. Although a full detector simulation as well as a thorough study of relevant kinematical cuts lies beyond the scope of this study, we nevertheless suggest several possibilities for kinematical cuts.

Instead of scanning over the parameter space of minimal supergravity (mSUGRA) inspired constrained SUSY models we illustrate our study via four representative benchmark points, both in the low and high $\tan \beta$ regime. ${ }^{1}$ In addition to complying with current LHC bounds, the chosen benchmark points exhibit a viable dark matter relic density [115]. Our analysis reveals that, despite the SM and SUSY backgrounds, the expected number of signal events should allow to probe cLFV in extensive regions of the SUSY seesaw parameter space.

The paper is organised as follows: in section 2, we briefly review Lepton Flavour Violation in the type I SUSY seesaw; sections 3 and 4 are respectively devoted to the discussion of a charged lepton flavour violation signal (and corresponding background) at a Linear Collider for the $e^{+} e^{-}$and $e^{-} e^{-}$beam options. In section 5 , we collect the relevant numerical results and discuss the potential observation of a SUSY seesaw cLFV signature at a Linear Collider, considering different beam configurations/polarisations, always stressing the correlation with the corresponding relevant low-energy observables. Our final remarks are given in section 6 . Technical details concerning the computation are summarised in the appendix.

\section{LFV in the SUSY seesaw}

Irrespective of the Dirac/Majorana nature of the neutrinos, and of the underlying mechanism of $\nu$-mass generation, charged lepton currents (i.e., $W^{ \pm} \bar{\ell}_{i} \nu_{j}$ ) do violate lepton flavour, with a strength given by the relevant entry of the $U_{\text {MNS }}$ leptonic mixing matrix, $U_{i j}$. Under the standard parametrization (and omitting Majorana CP violating phases for simplicity),

\footnotetext{
${ }^{1}$ Most of the chosen benchmark points comply with recent LHCb bounds on $\operatorname{BR}\left(B_{s} \rightarrow \mu^{+} \mu^{-}\right)[114]$.
} 
the $U_{\mathrm{MNS}}$ is given by

$$
U_{\mathrm{MNS}}=\left(\begin{array}{ccc}
c_{12} c_{13} & s_{12} c_{13} & s_{13} e^{-i \delta} \\
-s_{12} c_{23}-c_{12} s_{23} s_{13} e^{i \delta} & c_{12} c_{23}-s_{12} s_{23} s_{13} e^{i \delta} & s_{23} c_{13} \\
s_{12} s_{23}-c_{12} c_{23} s_{13} e^{i \delta} & -c_{12} s_{23}-s_{12} c_{23} s_{13} e^{i \delta} & c_{23} c_{13}
\end{array}\right),
$$

where $c_{i j} \equiv \cos \theta_{i j}, s_{i j} \equiv \sin \theta_{i j}$, with $\theta_{i j}$ the leptonic mixing angles and $\delta$ the Dirac CP phase.

Depending on the hypothesised mechanism of neutrino mass generation, and on the specific framework onto which it is embedded, additional sources of charged lepton flavour violation might emerge, leading to potentially important contributions to a number of cLFV observables.

Here we consider a type I seesaw embedded into the constrained MSSM (cMSSM), which is thus extended by three right-handed neutrino superfields. The model is defined by its superpotential and soft-SUSY breaking Lagrangian, whose leptonic parts we detail here:

$$
\mathcal{W}^{\text {lepton }}=\hat{N}^{c} Y^{\nu} \hat{L} \hat{H}_{2}+\hat{E}^{c} Y^{l} \hat{L} \hat{H}_{1}+\frac{1}{2} \hat{N}^{c} M_{N} \hat{N}^{c},
$$

where, and without any loss of generality, we work in a basis where both $Y^{l}$ and $M_{N}$ are diagonal, $Y^{l}=\operatorname{diag}\left(Y^{e}, Y^{\mu}, Y^{\tau}\right), M_{N}=\operatorname{diag}\left(M_{N_{1}}, M_{N_{2}}, M_{N_{3}}\right)$. The relevant slepton soft-breaking terms are then

$$
\begin{aligned}
\mathcal{V}_{\text {soft }}^{\text {slepton }}=-\mathcal{L}^{\text {slepton }}= & m_{\tilde{L}}^{2} \tilde{\ell}_{L} \tilde{\ell}_{L}^{*}+m_{\tilde{E}}^{2} \tilde{\ell}_{R} \tilde{\ell}_{R}^{*}+m_{\tilde{\nu}_{R}}^{2} \tilde{\nu}_{R} \tilde{\nu}_{R}^{*} \\
& +\left(A_{l} H_{1} \tilde{\ell}_{L} \tilde{\ell}_{R}^{*}+A_{\nu} H_{2} \tilde{\nu}_{L} \tilde{\nu}_{R}^{*}+B_{\nu} \tilde{\nu}_{R} \tilde{\nu}_{R}+\text { H.c. }\right) .
\end{aligned}
$$

Further assuming a flavour blind mechanism of SUSY breaking (for instance minimal supergravity inspired), the soft breaking parameters obey universality conditions at some highenergy scale, which we choose to be the gauge coupling unification scale $M_{\mathrm{GUT}} \sim 10^{16} \mathrm{GeV}$ :

$$
\begin{aligned}
\left(m_{\tilde{L}}\right)_{i j}^{2} & =\left(m_{\tilde{E}}\right)_{i j}^{2}=\left(m_{\widetilde{\nu}_{R}}\right)_{i j}^{2}=m_{0}^{2} \delta_{i j}, \\
\left(A_{l}\right)_{i j} & =A_{0}\left(Y^{l}\right)_{i j}, \quad\left(A_{\nu}\right)_{i j}=A_{0}\left(Y^{\nu}\right)_{i j},
\end{aligned}
$$

where $m_{0}$ and $A_{0}$ are the universal scalar soft-breaking mass and trilinear couplings of the cMSSM, and $i, j$ denote lepton flavour indices $(i, j=1,2,3)$.

After electroweak symmetry breaking (EWSB), and assuming the seesaw limit (i.e. $M_{N_{i}} \gg M_{\mathrm{EW}}$ ), one recovers the usual seesaw equation for the light neutrino mass matrix,

$$
m_{\nu}=-m_{D}^{\nu T} M_{N}^{-1} m_{D}^{\nu}
$$

with $m_{D}^{\nu}=Y^{\nu} v_{2}\left(v_{i}\right.$ being the vacuum expectation values (VEVs) of the neutral Higgs scalars, $v_{1(2)}=v \cos (\sin ) \beta$, with $\left.v=174 \mathrm{GeV}\right)$, and where $M_{N_{i}}$ corresponds to the masses of the heavy right-handed neutrino eigenstates. The light neutrino matrix $m_{\nu}$ is diagonalized by the $U_{\mathrm{MNS}}$ as $m_{\nu}^{\text {diag }}=U_{\mathrm{MNS}}{ }^{T} m_{\nu} U_{\mathrm{MNS}}$.

A convenient means of parametrizing the neutrino Yukawa couplings, while at the same time allowing to accommodate the experimental data, is given by the Casas-Ibarra parametrization [23], which reads at the seesaw scale, $M_{N}$,

$$
Y^{\nu} v_{2}=m_{D}^{\nu}=i \sqrt{M_{N}^{\text {diag }}} R \sqrt{m_{\nu}^{\text {diag }}} U_{\mathrm{MNS}^{\dagger}}^{\dagger}
$$


In the above, $R$ is a complex orthogonal $3 \times 3$ matrix that encodes the possible mixings involving the right-handed neutrinos, and which can be parameterized in terms of three complex angles $\theta_{i}(i=1,2,3)$.

The non-trivial flavour structure of $Y^{\nu}$ at the GUT scale will induce (through the running from $M_{\mathrm{GUT}}$ down to the seesaw scale) flavour mixing in the otherwise approximately flavour conserving soft-SUSY breaking terms. In particular, there will be radiatively induced flavour mixing in the slepton mass matrices, manifest in the $L L$ and $L R$ blocks of the $6 \times 6$ slepton mass matrix; an analytical estimation using the leading order (LLog) approximation leads to the following corrections to the slepton mass terms:

$$
\begin{aligned}
\left(\Delta m_{\tilde{L}}^{2}\right)_{i j} & =-\frac{1}{8 \pi^{2}}\left(3 m_{0}^{2}+A_{0}^{2}\right)\left(Y^{\nu \dagger} L Y^{\nu}\right)_{i j}, \\
\left(\Delta A_{l}\right)_{i j} & =-\frac{3}{16 \pi^{2}} A_{0} Y_{i j}^{l}\left(Y^{\nu \dagger} L Y^{\nu}\right)_{i j}, \\
\left(\Delta m_{\tilde{E}}^{2}\right)_{i j} & \simeq 0 ; \quad L_{k l} \equiv \log \left(\frac{M_{\mathrm{GUT}}}{M_{N_{k}}}\right) \delta_{k l} .
\end{aligned}
$$

The amount of flavour violation is encoded in the matrix elements $\left(Y^{\nu \dagger} L Y^{\nu}\right)_{i j}$ of eq. (2.7), which can be related to high- and low-energy neutrino parameters using eq. (2.6).

These renormalisation group equation (RGE) induced flavour violating corrections have an impact regarding both flavour non-universality and flavour violation in the charged slepton sector, leading to high- and low-energy cLFV observables that can be experimentally probed.

At low-energies, there will be important contributions to radiative and three-body lepton decays, as well as to $\mu-e$ conversion in nuclei (a detailed discussion, and the corresponding expressions can be found, for example, in [116]). In particular, and considering a seesaw scale close to the GUT scale (i.e. $\left.M_{N} \sim \mathcal{O}\left(10^{12-15} \mathrm{GeV}\right)\right)$, so that $Y^{\nu}$ is large $\left(Y^{\nu} \sim \mathcal{O}(1)\right)$, one expects values of $\operatorname{BR}(\mu \rightarrow e \gamma), \operatorname{BR}(\tau \rightarrow \mu \gamma)$ and $\operatorname{CR}(\mu-e, \mathrm{~N})$ to be well within the reach of current dedicated experiments.

At the LHC, cLFV can also be studied, for instance in relation with the $\chi_{2}^{0} \rightarrow \chi_{1}^{0} \ell^{ \pm} \ell^{\mp}$ decay chains. In scenarios where the $\chi_{2}^{0}$ is sufficiently heavy to decay via a real (on-shell) slepton, several cLFV observables can be manifest: (i) sizable widths for cLFV decay processes like $\chi_{2}^{0} \rightarrow \chi_{1}^{0} \ell_{i}^{ \pm} \ell_{j}^{\mp}[40-42,44,45]$; (ii) multiple edges in di-lepton invariant mass distributions $\chi_{2}^{0} \rightarrow \chi_{1}^{0} \ell_{i}^{ \pm} \ell_{i}^{\mp}$, arising from the exchange of a different flavour slepton $\tilde{\ell}_{j}$ (in addition to the left- and right-handed sleptons, $\tilde{\ell}_{L, R}^{i}$ ); (iii) flavoured slepton mass splittings.

As discussed, for instance in [46, 47, 49, 50], the interplay of low- and high-energy observables might provide valuable insight into the underlying mechanism of neutrino mass generation.

\section{LFV at a Linear Collider: $e^{+} e^{-}$beam option}

In this work we focus our attention on possible signals of cLFV in processes of the type $e^{+} e^{-} \rightarrow \ell_{i}^{+} \ell_{j}^{-}+$missing energy. It is important to emphasise here that one must distinguish LFV as originating from charged current interactions, and whose source is strictly related 
to the $U_{\text {MNS }}$ matrix (in a model-independent way), from LFV originating from neutral currents involving sleptons. The latter, although also associated to the $U_{\mathrm{MNS}}$, can be related to the implementation of a specific mechanism for cLFV: in [77], effective flavour violation entries in the slepton mass matrices were considered; in [78, 79], lepton flavour violation has been addressed for a Linear Collider in the framework of a type I SUSY seesaw. Here, and following the latter approach, we revisit cLFV arising from a type I SUSY seesaw at a Linear Collider in the light of recent neutrino data, low-energy cLFV bounds and, more importantly, for a comparatively heavy SUSY spectrum as suggested by recent LHC searches.

In our work, we will thus study signals of SUSY seesaw cLFV as

$$
e^{+} e^{-} \rightarrow \ell_{i}^{+} \ell_{j}^{-}+2 \chi_{1}^{0},
$$

further focusing on final states of the type $e^{+} \mu^{-}$, so to explore the maximal efficiency of the muon detector $[56,57,59]$. We recall that we do allow for (intermediate) tau production, but consider only its purely leptonic decays, $\tau \rightarrow \nu_{\tau} \ell \bar{\nu}_{\ell}$. Clearly, under the assumption of leptonic mixing, as parametrized by the $U_{\mathrm{MNS}}$, both the SM and the MSSM provide important, if not dominant, contributions. In particular, one expects missing energy signatures such as neutrinos pairs (in the case of the SM) and/or pairs of neutrinos +2 neutralinos in the case of the MSSM.

Before proceeding, let us mention that LFV can also be manifest in more involved sparticle production and decays: depending on the specific SUSY spectrum, other processes might be present, leading to more complex final states (e.g., with four charged leptons, mesons, and missing energy).

In this study we focus on the following processes

$$
e^{+} e^{-} \rightarrow\left\{\begin{array}{l}
e^{+} \mu^{-}+2 \chi_{1}^{0} \\
e^{+} \mu^{-}+2 \chi_{1}^{0}+(2,4) \nu \\
e^{+} \mu^{-}+(2,4) \nu
\end{array}\right.
$$

as well as the charge-conjugated final states.

The process (A), which corresponds to the signal, will arise from both $s$ - and $t$-channel processes. In the former case, one can have photon-, $Z$, and Higgs mediated production of slepton pairs (whose flavour content is decorrelated from the initial state electrons) and mixed neutralino $\left(\chi_{i}^{0} \chi_{j}^{0}\right)$ pair production. In the latter case, the $t$-channel exchange of neutralinos gives rise to slepton production. Although in most cases the event is expected to have a symmetric topology, it is important to notice that, if allowed by the SUSY spectrum, one might be led to asymmetric cases. Some illustrative examples of diagrams leading to final state of type (A) can be found in figure 1 .

(B) encompasses most of the processes leading to what we denote "charged current SUSY background", in other words cLFV processes that occur via SUSY charged currents. In this case, flavour violation reflects only the existence of low-energy leptonic mixings, and could even be accommodated with massive Dirac neutrinos. As an example, one can have mixed chargino $\left(\chi_{1,2}^{ \pm} \chi_{1,2}^{\mp}\right)$ s-channel production, while gauginos can also be produced via 


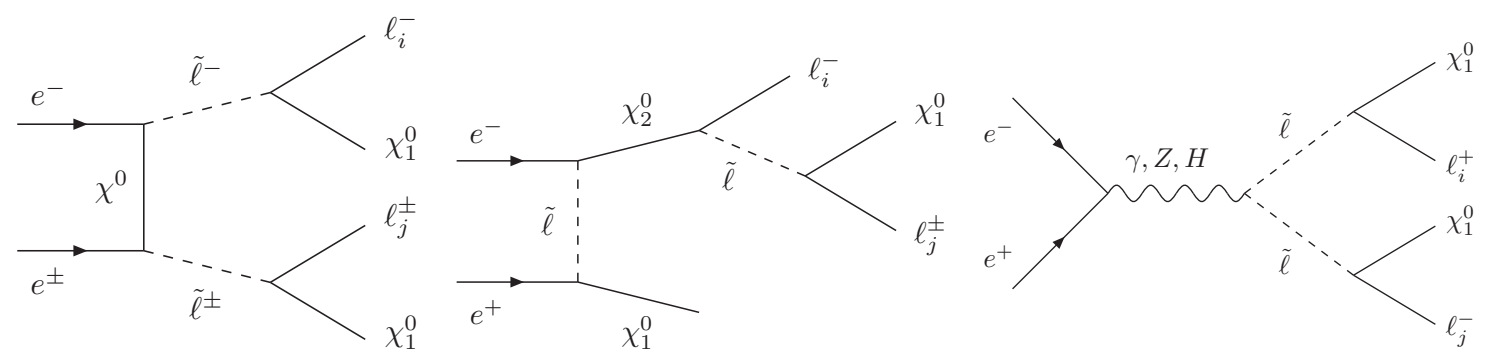

Figure 1. Illustrative examples of processes contributing to signal (A), for both $e^{+} e^{-}$and $e^{-} e^{-}$ beam options.

the $t$-channel exchange of a neutral or charged slepton. Moreover, the decays of a would-be final state tau (i.e., an intermediate tau state) can account for additional neutrino pairs.

Finally, the SM background (processes of type (C)) corresponds to $s$-channel photon exchange, and/or $t$-channel lepton exchange (Dirac or Majorana neutrinos). Like for the SUSY background, " $W$-strahlung" can lead to sizable contributions. Different flavour opposite-sign final state leptons would arise from charged current interactions and, as in processes of type (B), would only reflect the existence of low-energy mixings in the lepton sector. Multiple final state neutrino pairs can arise, for instance, in association to intermediate tau decays (as in (B)).

Examples of background processes of type (B) and (C) can be found in figures 2 and 3. Notice that some of the processes displayed are due to the nature of the light neutrinos (Dirac vs. Majorana), while the viability of others is dictated by the specific SUSY spectra.

We notice that the distinct topology of the different processes, as well as specific dedicated cuts, should already allow a first selection of the "signal" events (A).

The possibility of electron and positron beam polarisation is also instrumental in disentangling the above processes [64,65]: in the ideal case of $100 \%$ polarised $e^{+}$and $e^{-}$ beams, that is an LL polarisation of $\left(P_{e^{-}}, P_{e^{+}}\right)=(-100 \%,-100 \%)$, and with the exception of the $W$-strahlung processes, the other SM contributions (C) would vanish, as well as most of those arising from pure SUSY LFV charged currents (B). Under this "ideal" beam configuration, and via some kinematical cuts, a cLFV signal (A) would clearly point towards flavour mixing in the slepton sector (for instance due to a SUSY seesaw), manifest in neutral currents.

Other sources of background have also been considered. For instance, the Higgsmediated contributions have been taken into account, but their contributions to final states of the type $e^{+} \mu^{-}+(0$ or 4$) \chi_{1}^{0}+(2,4$ or 6$) \nu$ are small, especially compared to the previously discussed processes $(\mathrm{C})$. Likewise, three body processes such as $e^{+} e^{-} \rightarrow$ $\chi^{0} \ell^{ \pm} \tilde{\ell}^{\mp}, \chi^{ \pm} W^{\mp} \chi^{0}$ and $\tilde{\ell}^{ \pm} W^{\mp} \tilde{\nu}$ are found to be subdominant and have thus been neglected.

A final remark, concerning the possibility of same-flavour final states (such as $\mu^{+} \mu^{-}$) is still in order. Although same-flavour final state leptons will be explored in the case of the $e^{-} e^{-}$beam option (in sections 4 and 5.3), we will not consider them here, since such a signal would be dominantly generated from flavour conserving interactions. 

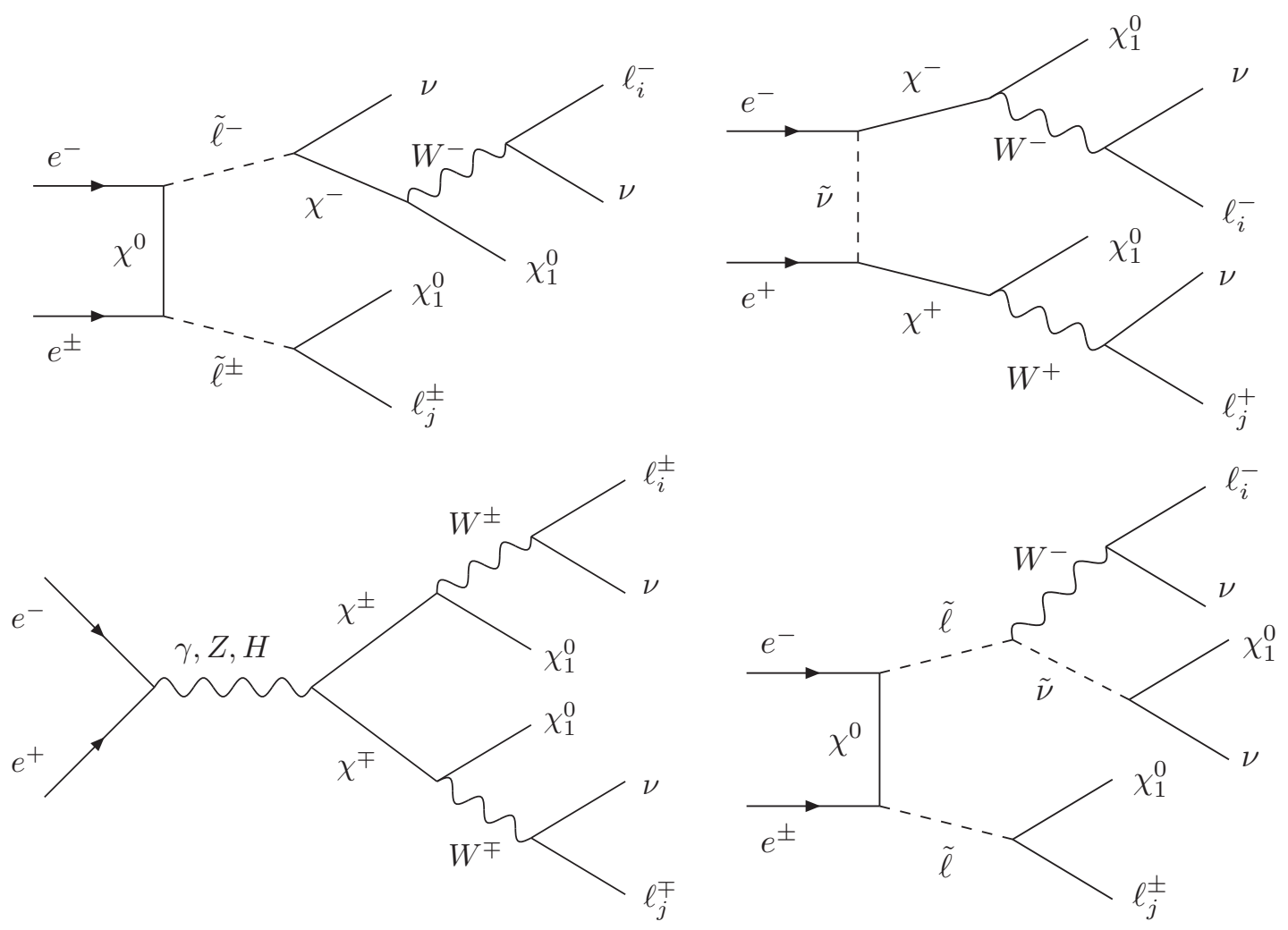

Figure 2. Examples of processes contributing to the SUSY background (B), for both $e^{+} e^{-}$and $e^{-} e^{-}$beam options.

\section{$4 \quad$ LFV at a Linear Collider: $e^{-} e^{-}$beam option}

In addition to its many potentialities to probe the Majorana nature of neutral fermions which implies the violation of total lepton number, $e^{-} e^{-}$collisions provide one of the cleanest experimental environments to test lepton flavour violation in the slepton sector [78]. In the SM and the MSSM (and their type I seesaw extensions), s-channel processes are excluded due to the absence of doubly charged particles (for instance, as those present in a type II seesaw [9-15]). As done in the previous section, we again focus on $e^{-} e^{-} \rightarrow \ell_{i}^{-} \ell_{j}^{-}+E_{\text {miss }}^{T}$ (at least $i$ or $j \neq e$ ). As for the $e^{+} e^{-}$case, we include processes involving intermediate $\tau \mathrm{s}$, which decay only leptonically, as background contributions.

In particular, we study the following possibilities:

$$
e^{-} e^{-} \rightarrow\left\{\begin{array}{l}
e^{-} \mu^{-}+2 \chi_{1}^{0} \\
e^{-} \mu^{-}+2 \chi_{1}^{0}+(2,4) \nu \\
e^{-} \mu^{-}+(2,4) \nu
\end{array}\right.
$$

Some diagrams corresponding to these processes were illustrated in figures 1, 2 and 3.

In the processes of type $(\mathrm{A})$ - signal - , charged slepton production occurs via $t$ channel neutralino exchange. For SUSY spectra in which $m_{\tilde{\ell}} \lesssim m_{\chi_{1}^{ \pm}}$, this is typically the dominant channel leading to $\ell_{i}^{-} \ell_{j}^{-}+2 \chi_{1}^{0}$ final states. 

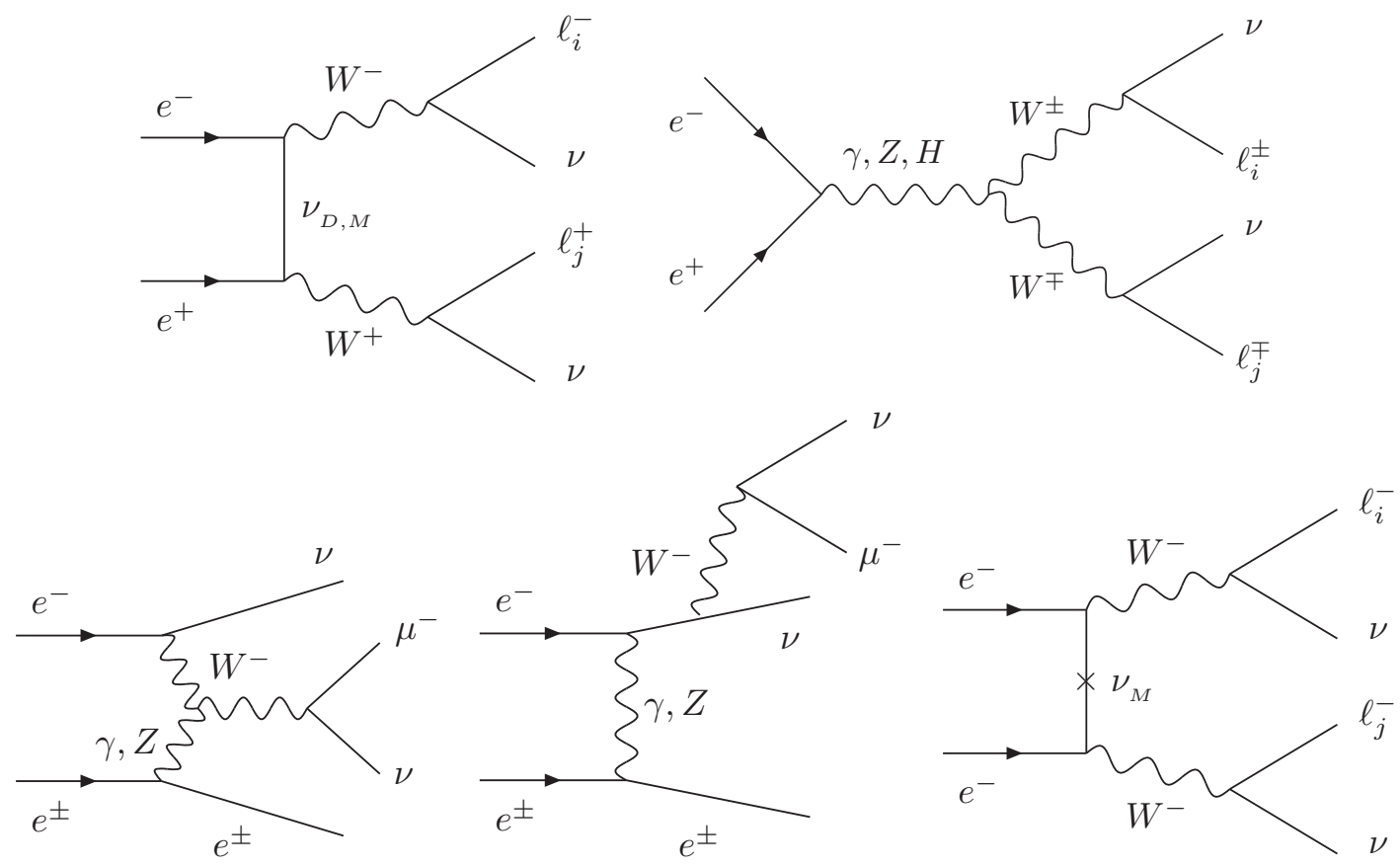

Figure 3. Examples of processes contributing to the SM background (C), for both $e^{+} e^{-}$and $e^{-} e^{-}$ beam options.

Other than the contributions coming from single $W$-production ( $W$-strahlung), we notice that additional processes might contribute to the background. For example, if the spectrum is such that $m_{\tilde{\ell}} \gtrsim m_{\chi_{1}^{ \pm}}$, one of the sleptons decays via $\tilde{\ell}^{-} \rightarrow \nu \chi_{1}^{-} \rightarrow \nu \chi_{1}^{0} W^{-} \rightarrow$ $2 \nu \chi_{1}^{0} \ell^{-}$. We stress that in this case, polarising the beams has little effect on disentangling the signal (A) from the background (B), as both processes occur via identical slepton production ( $t$-channel neutralino exchange) mechanisms.

The SM background (C) would arise from $t$-channel single $W$-production, as well as from the exchange of a Majorana neutrino; notice that the latter cross section (and similar to what occurs for neutrinoless double beta decays $-0 \nu 2 \beta-$ ) is extremely tiny, at most $\sigma\left(e^{-} e^{-} \rightarrow W^{-} W^{-}\right) \sim \mathcal{O}\left(10^{-22} \mathrm{fb}\right)$, due to the smallness of the light neutrino masses.

In addition to studying the prospects for $e^{-} \mu^{-}$final states, a $\mu^{-} \mu^{-}+$missing energy final state is a truly "golden channel" for cLFV at a Linear Collider. In this case, the SM background $(\mathrm{C})$ is negligible, and the SUSY background processes are always subdominant. Furthermore, in addition to probing cLFV in neutral currents in a remarkably clean way, this channel also allows to fully benefit from the LC muon detector efficiency.

\section{LC potential for LFV discovery}

We now proceed to address the different cLFV processes discussed in the previous sections (eqs. (3.2), (4.1)), considering that the unique source of LFV is a type I seesaw mechanism.

As discussed in section 2, we embed a type I seesaw into the framework of the cMSSM. At the GUT scale, the neutrino Yukawa couplings are parametrized as in eq. (2.6), and 


\begin{tabular}{|c|c|c|c|c|}
\hline & C-light & C-heavy & F-light & F-heavy \\
\hline$m_{0}(\mathrm{GeV})$ & 150 & 200 & 600 & 750 \\
\hline$M_{1 / 2}(\mathrm{GeV})$ & 727.9 & 949.2 & 667.0 & 872.1 \\
\hline $\tan \beta$ & 10 & 10 & 52 & 52 \\
\hline$A_{0}(\mathrm{GeV})$ & 0 & 0 & 0 & 0 \\
\hline $\operatorname{sign}(\mu)$ & 1 & 1 & 1 & 1 \\
\hline
\end{tabular}

Table 1. Representative points used in the numerical analysis.

we adopt a conservative approach where no mixings, other than those associated to the $U_{\mathrm{MNS}}$, are present ${ }^{2}$ (i.e. $R=1$ ). Concerning low-energy lepton data, we take the following (best-fit) values for the neutrino mixing angles (solar and atmospheric) [83, 117],

$$
\sin ^{2} \theta_{12}=0.31_{-0.015}^{+0.017}, \quad \sin ^{2} \theta_{23}=0.52_{-0.07}^{+0.06} .
$$

In what concerns the Chooz angle, given the very recent results from Double-Chooz [84], T2K [85], MINOS [86], Daya-Bay [87] and RENO [88], we choose a representative value for this experimentally favoured large $\theta_{13}$ regime:

$$
\theta_{13}=10^{\circ} \text {. }
$$

Finally, the light neutrino spectrum is specified by the following intervals for the masssquared differences

$$
\Delta m_{12}^{2}=(7.6 \pm 0.2) \times 10^{-5} \mathrm{eV}^{2}, \quad \Delta m_{13}^{2}=\left\{\begin{array}{l}
(-2.40 \pm 0.09) \times 10^{-3} \mathrm{eV}^{2} \\
(+2.50 \pm 0.13) \times 10^{-3} \mathrm{eV}^{2}
\end{array}\right.
$$

where the two ranges for $\Delta m_{13}^{2}$ correspond to normal and inverted neutrino spectrum [83].

Although in our study we considered both hierarchical and degenerate right-handed neutrino spectra, in the following discussion we will present the results for the case of a degenerate right-handed neutrino spectrum.

In our numerical analysis, and to illustrate the LFV potential of a Linear Collider, we will consider two sets of cMSSM (mSUGRA-like) points, ${ }^{3}$ defined in table 1 , representative of regimes of low and (very) large $\tan \beta$. For each regime, we further present two possibilities, one with a "light" spectrum (i.e. with a gluino mass just above the LHC exclusion bound) and the other with a comparatively heavier one (associated with $m_{\tilde{g}}=2 \mathrm{TeV}$ ). The points with low $\tan \beta$ have a phenomenology similar to that of the points along the 10.1.n slope of the recent LHC benchmark proposal [119]. Moreover, the relatively lowvalues of $m_{0}$ (when compared to $M_{1 / 2}$ ) lead to a lighter slepton spectrum, that can be easily produced at a LC (while avoiding the LHC bounds on the strongly interacting sector). We notice that in this case, the correct dark matter relic density (computed using

\footnotetext{
${ }^{2}$ In general, the limit $R=1$ translates into a "conservative" limit for flavour violation: apart from possible cancellations, and for a fixed seesaw scale, this limit typically provides a lower bound for the amount of the generated LFV.

${ }^{3}$ In the final stages of this work, a new ILC-dedicated benchmark proposal has appeared [118].
} 


\begin{tabular}{|c|c|c|c|c|}
\hline & C-light & C-heavy & F-light & F-heavy \\
\hline$\chi_{1}^{0}$ & 303.7 & 401.1 & 279.9 & 370.6 \\
\hline$\chi_{2}^{0}$ & 574.2 & 756.9 & 530.3 & 700.9 \\
\hline$\chi_{3}^{0}$ & -882.3 & -1114.7 & -786.5 & -993.9 \\
\hline$\chi_{4}^{0}$ & 893.5 & 1124.4 & 797.5 & 1003.8 \\
\hline$\chi_{1}^{ \pm}$ & 574.4 & 757.1 & 530.5 & 701.1 \\
\hline$\chi_{2}^{ \pm}$ & 893.8 & 1124.5 & 798.3 & 1004.2 \\
\hline$\left(\tilde{\nu}_{e}\right)_{L}$ & 504.2 & 657.9 & 742.4 & 943.3 \\
\hline$\left(\tilde{\nu}_{\mu}\right)_{L}$ & 503.9 & 657.6 & 651.0 & 830.0 \\
\hline$\left(\tilde{\nu}_{\tau}\right)_{L}$ & 502.1 & 655.2 & 741.8 & 368.93 \\
\hline$\tilde{e}_{R}$ & 314.4 & 409.7 & 650.3 & 817.6 \\
\hline$\tilde{e}_{L}$ & 510.7 & 662.9 & 747.0 & 946.9 \\
\hline$\tilde{\mu}_{R}$ & 314.4 & 409.7 & 649.5 & 816.6 \\
\hline$\tilde{\mu}_{L}$ & 509.9 & 662.6 & 746.5 & 946.3 \\
\hline$\tilde{\tau}_{1}$ & 306.5 & 401.1 & 383.6 & 495.8 \\
\hline$\tilde{\tau}_{2}$ & 510.6 & 661.4 & 672.8 & 847.4 \\
\hline
\end{tabular}

Table 2. Slepton, neutralino and chargino mass spectrum (in $\mathrm{GeV}$ ) for the points of table 1 .

MicrOmegas [120]) is obtained from neutralino-stau co-annihilation, and these points are thus characterised by a very small NLSP-LSP mass difference. The very large value of $\tan \beta=52$ chosen for the second set of points is also due to our willingness to have the LSP as a viable dark matter candidate (in this case we are in the so-called "Higgs funnel" region).

In table 2, we collect the most relevant information regarding the spectrum of the chosen benchmark points. The low-energy sparticle masses and mixings have been numerically evaluated with the SPheno public code [121]. LHC bounds on the SUSY spectrum [89-107] have been applied; in all cases, the lightest Higgs mass is around $117 \mathrm{GeV}$, still marginally compatible with the new LHC data $[122,123]$. Notice that the non-minimal SUSY scenarios can still be considered to accommodate such data, with a minimal impact concerning the present lepton flavour dedicated analysis. This is the case of deviations from strict mSUGRA inspired universality in the Higgs sector - the so-called Non-Universal Higgs Mass models - , or third generation non-universality, which could increase the value of $m_{h}$ (especially given the already heavy stop sector) [124].

We have taken into account all relevant constraints from low-energy cLFV dedicated experiments [108]. In particular, regarding the bound on $\operatorname{BR}(\mu \rightarrow e \gamma)$, which will be the most constraining one for our study, we have considered the most recent MEG results [109],

$$
\operatorname{BR}(\mu \rightarrow e \gamma)<2.4 \times 10^{-12}, \quad 90 \% \text { C.L. . }
$$

A comment is still in order concerning the recent $\mathrm{LHCb}$ bounds of $\operatorname{BR}\left(B_{s} \rightarrow \mu^{+} \mu^{-}\right)$[114]: points $\mathrm{F}$ (in the large $\tan \beta$ regime) are associated with large contributions to the latter 
observable. In particular due to its lighter spectrum, F-light is clearly ruled out based on this bounds, while F-heavy would be marginally compatible at $3-\sigma$. Both points $\mathrm{C}$ are in good agreement with these bounds, and in the subsequent discussion we will mostly illustrate our results focusing on points C-light and C-heavy.

In what follows, we separately discuss the prospects for $e^{+} e^{-}$and $e^{-} e^{-}$, and in both cases the effects of polarising the beams. In our analysis, we have taken into account all contributions to the different final states, allowing for the production and exchange of all possible (s-)states. ${ }^{4}$ Our study is based on an algorithmic calculation of the possible production and decay modes, considering that the majority of the events proceeds from an on-shell primary production (so that there are no interference effects between the different contributions), with subsequent two-body cascade decays (the exception being 3-body decays of the $\tau$ ). For SUSY $e^{+} e^{-}$cross-sections and branching fractions, we have used SPheno [121], while for SUSY $e^{-} e^{-}$and SM cross sections we have developed dedicated routines. For each given final state, we have generated all possible allowed production and decay chains, arising from each of the considered primary production modes (more detailed information can be found in the appendix).

Regarding the performance of the Linear Collider, we will assume values for the c.o.m. energy in the interval $500 \mathrm{GeV} \lesssim \sqrt{s} \lesssim 3 \mathrm{TeV}$, and benchmark values for the integrated luminosity of 0.5 and $3 \mathrm{ab}^{-1}$. In our analysis, we adopt the following definition of the significance of the cLFV signal:

$$
\mathcal{S}=\frac{\mathcal{N}(\text { signal })}{\sqrt{\mathcal{N}(\text { signal }+ \text { background })}},
$$

where $\mathcal{N}$ denotes the number of events and "background" will be identified with (B) and/or $(\mathrm{B}+\mathrm{C})$, see eqs. (3.2), (4.1).

In this study we will only conduct a phenomenological analysis, i.e., focusing only on the theoretical estimations of the potential number of events at a $\mathrm{LC}$ operating at a given $\sqrt{s}$, for possibly polarised beams. Although Linear Collider CRDs do not consider cLFV slepton production and/or decays, there are dedicated studies addressing, for example in the case of $e^{+} e^{-} \rightarrow \tilde{\ell}^{+} \tilde{\ell}^{-} \rightarrow \ell_{i}^{+} \ell_{i}^{-} \chi_{1}^{0} \chi_{1}^{0}$, event reconstruction methods and detector performances, as well as proposals for several cuts that allow to optimise the significance. The different cuts are based on the key characteristics of the signal events which, in addition to the different flavour final state leptons, are missing energy, missing transverse momentum and acoplanarity. The analysis procedure proposed in [125] makes use of the following discriminating variables to separate the signal from the large backgrounds: dilepton energy $\left(E_{\ell_{i}}+E_{\ell_{j}}\right)$; vector and algebraic sums of the leptons' momenta $\left(p_{\ell_{i}}^{T}, p_{\ell_{j}}^{T}\right)$; dilepton invariant mass $m_{\ell_{i} \ell_{j}}$ and velocity $\beta_{\ell_{i} \ell_{j}}$; dilepton's missing momenta angle $\theta\left(\vec{p}_{\ell_{i}}^{T}, \vec{p}_{\ell_{j}}^{T}\right)$; dilepton acolinearity and acoplanarity; dilepton energy imbalance $\Delta=\left|E_{\ell_{i}}-E_{\ell_{j}}\right| /\left|E_{\ell_{i}}+E_{\ell_{j}}\right|$. The values of the above cuts are chosen to optimise significance versus signal efficiency. As discussed in [57], selection efficiencies can be as high as $97 \%$ for di-muon and $94 \%$ for di-electron

\footnotetext{
${ }^{4}$ Although in the present analysis we only collect the results regarding the leading-order LFV processes mentioned above, we have also investigated more complex production and decay processes, with additional LFV vertices and longer decay chains, leading to final states involving a larger number of particles.
} 
final states. Therefore based on the similarities between the flavour conserving processes discussed in $[57,125]$ with the cLFV signals studied here, we expect that a dedicated parallel analysis with the introduction of appropriate cuts will allow to optimise the statistical significance in the cLFV case as well. The LC appears thus a perfect facility for the study of $e^{ \pm} e^{-} \rightarrow e^{ \pm} \mu^{-}+E_{\text {miss }}^{T}$ and $e^{-} e^{-} \rightarrow \mu^{-} \mu^{-}+E_{\text {miss }}^{T}$ processes.

\section{$5.1 e^{+} e^{-}$beam option}

We begin by studying the processes $e^{+} e^{-} \rightarrow e^{+} \mu^{-}+E_{\text {miss }}^{T}$, with $E_{\text {miss }}^{T}=2 \chi_{1}^{0}, 2 \chi_{1}^{0}+$ $(2,4) \nu,(2,4) \nu$, for the four representative points described in table 1 (C-light, C-heavy, F-light and F-heavy). We display in figure 4 these cross section for points C-light and C-heavy, assuming a degenerate right-handed neutrino spectrum.

As can be easily seen from the left panels of figure 4, the SM background (in particular due to the $W$-strahlung processes) clearly dominates over any SUSY contribution, for all values of the c.o.m. energy, $\sqrt{s}$. However, due to the different topology of the SM background (C), when compared to the SUSY events (A) and (B), we expect (in view of what was previously argued) that these can be successfully disentangled (see for instance the several cuts above, as well as those proposed in [57]. Due to the very light spectrum of both C-points (see tables 1 and 2), the signal is associated to a slightly larger cross section, and for the case of C-heavy, it could be the only SUSY signal for low values of $\sqrt{s}$. Although not displayed here, we have also estimated the contributions of Higgs-mediated processes (via intermediate $h Z, H A$ or $H^{+} H^{-}$production), finding that these are very subdominant, with associated cross sections typically below $10^{-1} \mathrm{fb}$. Moreover, we notice that the contribution from intermediate $\tau$ decays accounts for about about $90 \%$ of the SUSY background in the case of points F, and circa $50 \%$ (100\% for LL polarization) in the case of points $\mathrm{C}$, provided the c.o.m. energy is above the $\tilde{\ell}_{L} \tilde{\ell}_{R}$ production threshold; otherwise it is about $100 \%$ for both cases.

Additional information, especially on the seesaw origin of the signal, can be found on the right-handside plots, where we display its dependence on the degenerate righthanded neutrino mass, $M_{R}$. For point C-heavy, the seesaw origin of the signal is manifest, increasing with $M_{R}$ as would be expected from the LLog approximation of eq. (2.7), thus revealing that such an $e^{+} e^{-} \rightarrow e^{+} \mu^{-}+2 \chi_{1}^{0}$ cross section is clearly due to the working hypothesis of a type I SUSY seesaw. For sufficiently large values of the seesaw scale, the signal (A) would even dominate over the SUSY background (B), but such regions become eventually excluded, as the associated amount of flavour violation in the $e-\mu$ sector would induce excessively large $\mathrm{BR}(\mu \rightarrow e \gamma)$. The peculiar spectrum of point C-light, with near-degenerate $\tilde{e}_{L}, \tilde{\mu}_{L}$ states, is such that even for a very small $L L$ slepton mixing (i.e. $\left.\left(m_{L L}^{\tilde{\ell}}\right)_{12}^{2} \ll\left(m_{L L}^{\tilde{\ell}}\right)_{11,22}^{2}\right)$, sufficiently small to avoid $\mu \rightarrow e \gamma$ constraints, a significant $\tilde{e}_{L}-\tilde{\mu}_{L}$ mixing can indeed occur due to tiny $L R$ effects. This accounts for the apparent "flatness" exhibited by the signal $\sigma$ versus $M_{R}$ curves.

Assuming an integrated luminosity of $0.5(3) \mathrm{ab}^{-1}$, the expected number of signal events would be $\mathcal{O}\left(5 \times 10^{3}\left(10^{4}\right)\right)$ for points C-light and $\mathcal{O}\left(10^{3}\left(10^{4}\right)\right)$ for C-heavy. In the former, the maximal number of events is associated with a lower regime of $M_{R}$, while in the latter, one finds the opposite behaviour (in agreement with figure 4). 

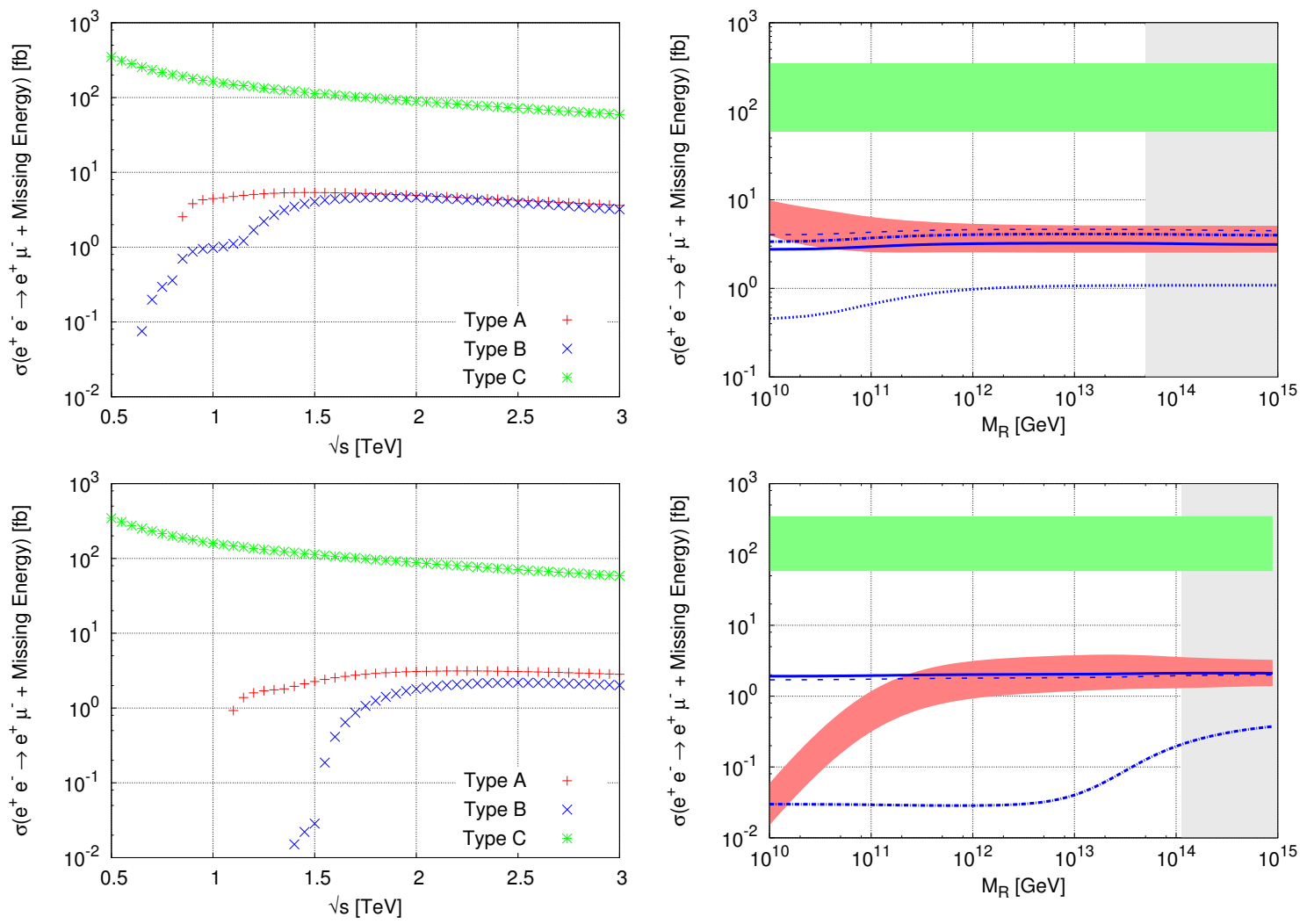

Figure 4. On the left, cross section for $e^{+} e^{-} \rightarrow e^{+} \mu^{-}+E_{\text {miss }}^{T}$ (with $E_{\text {miss }}^{T}=2 \chi_{1}^{0}, 2 \chi_{1}^{0}+$ $(2,4) \nu,(2,4) \nu)$, for points C-light and C-heavy (upper and lower panels, respectively), as a function of the centre of mass energy, $\sqrt{s}$. We fix $M_{R}=10^{12} \mathrm{GeV}$, and denote the signal (A) with red crosses, the SUSY charged current background (B) with blue times, and the SM charged current background (C) by green asterisks. On the right, cross section for $e^{+} e^{-} \rightarrow e^{+} \mu^{-}+E_{\text {miss }}^{T}$ (with $E_{\text {miss }}^{T}=2 \chi_{1}^{0}, 2 \chi_{1}^{0}+(2,4) \nu,(2,4) \nu$ ), for points C-light and C-heavy (upper and lower panels, respectively), as a function of the right-handed neutrino mass $\left(M_{R}\right)$. A green band denotes the SM background, while the red one corresponds to the signal (A). Blue lines denote the SUSY backgrounds, computed for $\sqrt{s}=1,1.5,2$ and $3 \mathrm{TeV}$ (respectively corresponding to dotted, dasheddotted, dashed and full lines). The (grey) shaded region corresponds to values of $M_{R}$ already excluded by the present bound on $\operatorname{BR}(\mu \rightarrow e \gamma)$. In all cases we have taken a degenerate right-handed neutrino spectrum, and set $\theta_{13}=10^{\circ}$.

In figure 5, we display analogous studies, but now for points F-light and F-heavy. The prospects are very similar to those of points $\mathrm{C}$, the only relevant difference being that for all experimentally compatible $M_{R}$ and all $\sqrt{s}$ regimes, the cross sections for the SUSY background (B) are much larger than those of the signal, (A). For the same nominal values of the integrated luminosity, the expected number of events would be however much smaller than for points $\mathrm{C}: \mathcal{O}(50(300))$ for F-light and $\mathcal{O}(10(100))$ for F-heavy, for $\mathcal{L}=0.5(3) \mathrm{ab}^{-1}$. Since points $\mathrm{F}$ are associated with excessively large contributions to $\operatorname{BR}\left(B_{s} \rightarrow \mu^{+} \mu^{-}\right)$, and thus experimentally disfavoured, we will focus most of the subsequent analysis on points C-light and C-heavy. 

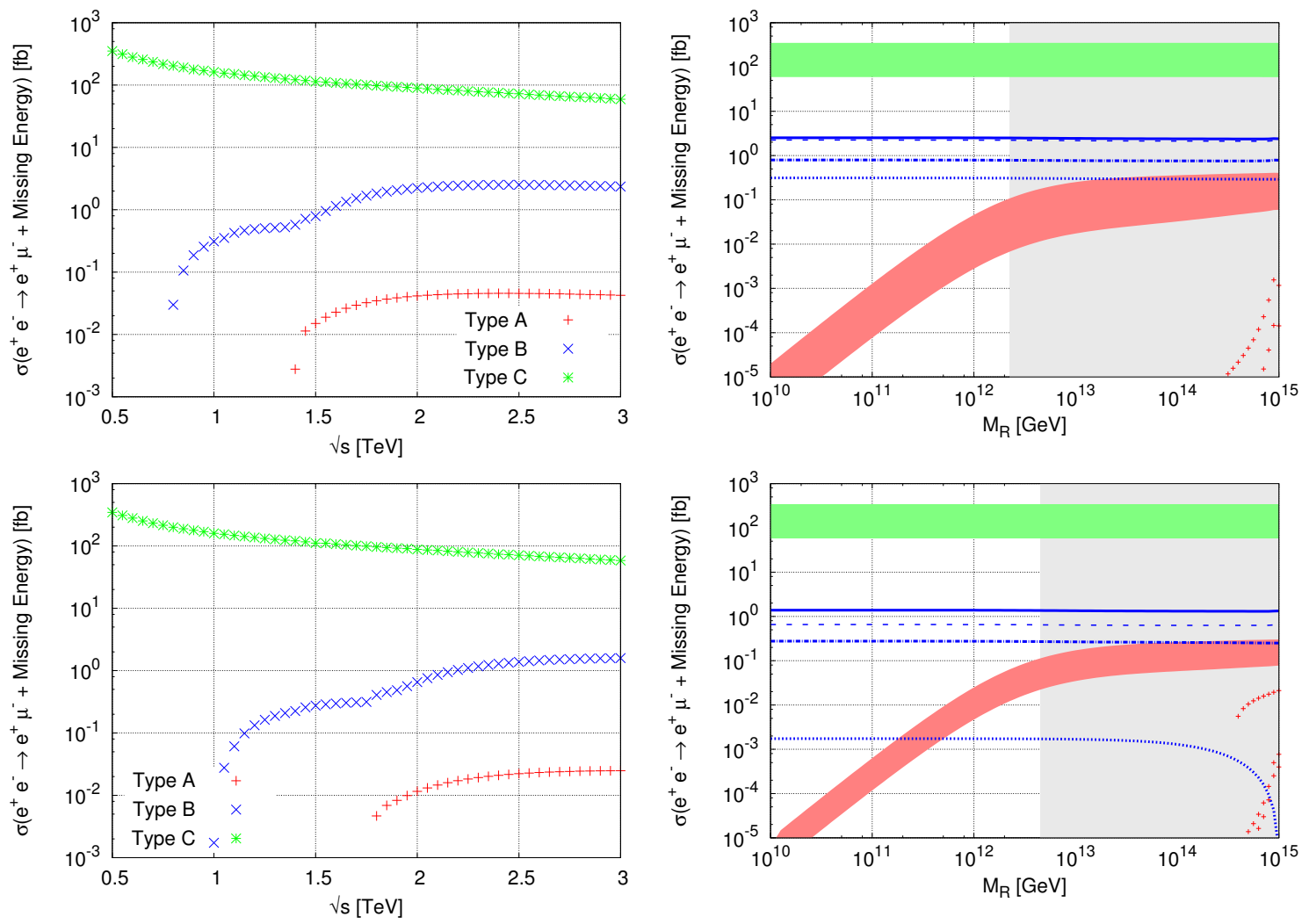

Figure 5. Same as in figure 4, but for points F-light and F-heavy (upper and lower panels, respectively), the only difference being that a full line is now associated to $\sqrt{s}=2.5 \mathrm{TeV}$.

For completeness, we display in figure 6 the significance of the cLFV seesaw signal (cf. eq. (5.5)) as a function of the seesaw scale, for points C-light and C-heavy. For illustrative purposes, we have chosen $\sqrt{s}=2 \mathrm{TeV}$. As is manifest, for the case of point C-light, one expects that the significance of the events should be no smaller than 20 (10) for the case of luminosity of $3(0.5) \mathrm{ab}^{-1}$, throughout the considered interval for $M_{R}$. By itself, and in the absence of dedicated cuts that would in principle allow to enhance the significance, this is an extremely promising result, in the sense that the observation of seesaw-induced cLFV events appears to be potentially feasible at a Linear Collider. As expected, the situation is slightly worse for a C-heavy spectrum; nevertheless, a significance close to 10 is potentially within reach for the considered integrated luminosities.

For both cases (points $\mathrm{C}$ and $\mathrm{F}$ ), considering a hierarchical RH neutrino spectrum does not have a significant impact on the results displayed before, other than the well-known effects on cLFV (see, e.g. [116]), such as a small reduction of the amount of flavour violation in the $e-\mu$ for the same choice of $M_{N_{3}}=M_{R}$.

Let us recall here that these results correspond to the case where there are no sources of flavour violation other than the $U_{\mathrm{MNS}}$, i.e. the limit $R=1$. This indeed corresponds to a "conservative" limit for flavour violation: apart from possible cancellations, and for a fixed seesaw scale, this limit typically provides a lower bound for the amount of the generated 

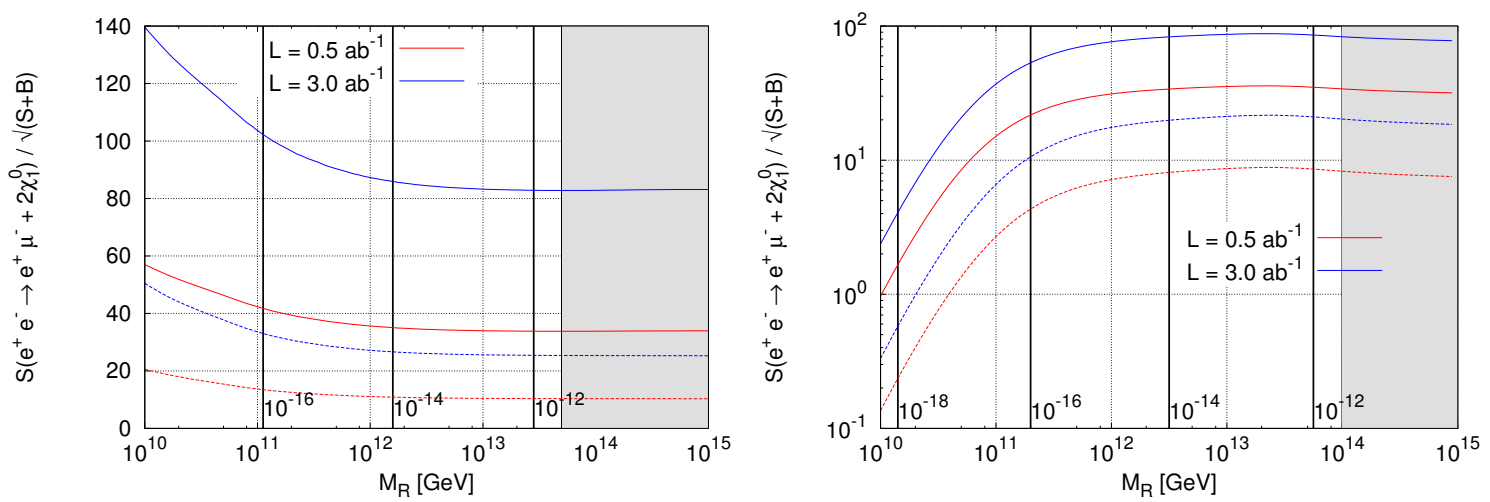

Figure 6. Significance of the signal for points C-light (left) and C-heavy (right), as a function of the seesaw scale $\left(M_{R}\right)$, for $\sqrt{s}=2 \mathrm{TeV}$. We assume nominal values for the integrated luminosity of 0.5 and $3 \mathrm{ab}^{-1}$ (respectively red and blue curves). The full/dashed lines correspond to a SUSY/SM + SUSY background, i.e., (B)/(B+C). Vertical lines denote the $M_{R^{-}}$corresponding value of $\mathrm{BR}(\mu \rightarrow e \gamma)$ while the (grey) shaded region represents values of $M_{R}$ already excluded by the present experimental bound on $\operatorname{BR}(\mu \rightarrow e \gamma)$. Same assumptions for the remaining parameters as in figure 4 .

LFV. For the present analysis, taking generic (complex) values ${ }^{5}$ of $R_{i j}$ would lead to an increase in the cross sections; however, this would also generate large contributions to low-energy cLFV observables (namely $\mu \rightarrow e \gamma$ ), strongly reducing the available parameter space.

We now consider how polarising the beams can help resolve the signal. As discussed before, if LFV charged currents - both arising from the SM background (C) and SUSY background (B) - can be eliminated, any remaining cLFV signal such as those of (A) would clearly point towards flavour mixing in the slepton sector that would be manifest in neutral currents.

In what follows, and in addition to the "ideal" polarisation scenario, i.e. a full LL polarisation of $\left(P_{e^{+}}, P_{e^{-}}\right)=(-100 \%,-100 \%)$, we consider a more realistic (but still optimistic) case where one could have $80 \%$ polarisation for both beams, $\left(P_{e^{+}}, P_{e^{-}}\right)=(-80 \%,-80 \%)$. In figure 7 we display the resulting cross sections, for points C-light and C-heavy. Notice that due to the $\mathrm{SU}(2)_{L}$ nature of the dominant $W$-strahlung background, LL polarisation cannot fully remove these contributions. Otherwise, polarising the electron and positron beams would significantly enhance the signal with respect to the remaining SM and other SUSY background. For instance, for point C-light, $80 \%$ beam polarisation would already allow to have a dominant cLFV signal, while in both $\mathrm{C}$ points, fully polarised beams would reduce the background stemming from processes other than $W$-emission. If dedicated detector simulations - with appropriated cuts - can indeed reduce the SM background (especially the dominant contributions due to single- and double- $W$ emissions), and thus isolate the SUSY contributions, polarised electron and positron beams would allow to ob-

\footnotetext{
${ }^{5}$ We notice that for the case of degenerate right-handed neutrinos, any real $R$ matrix is equivalent to taking $R=1$, as can be seen from eq. (2.6).
} 

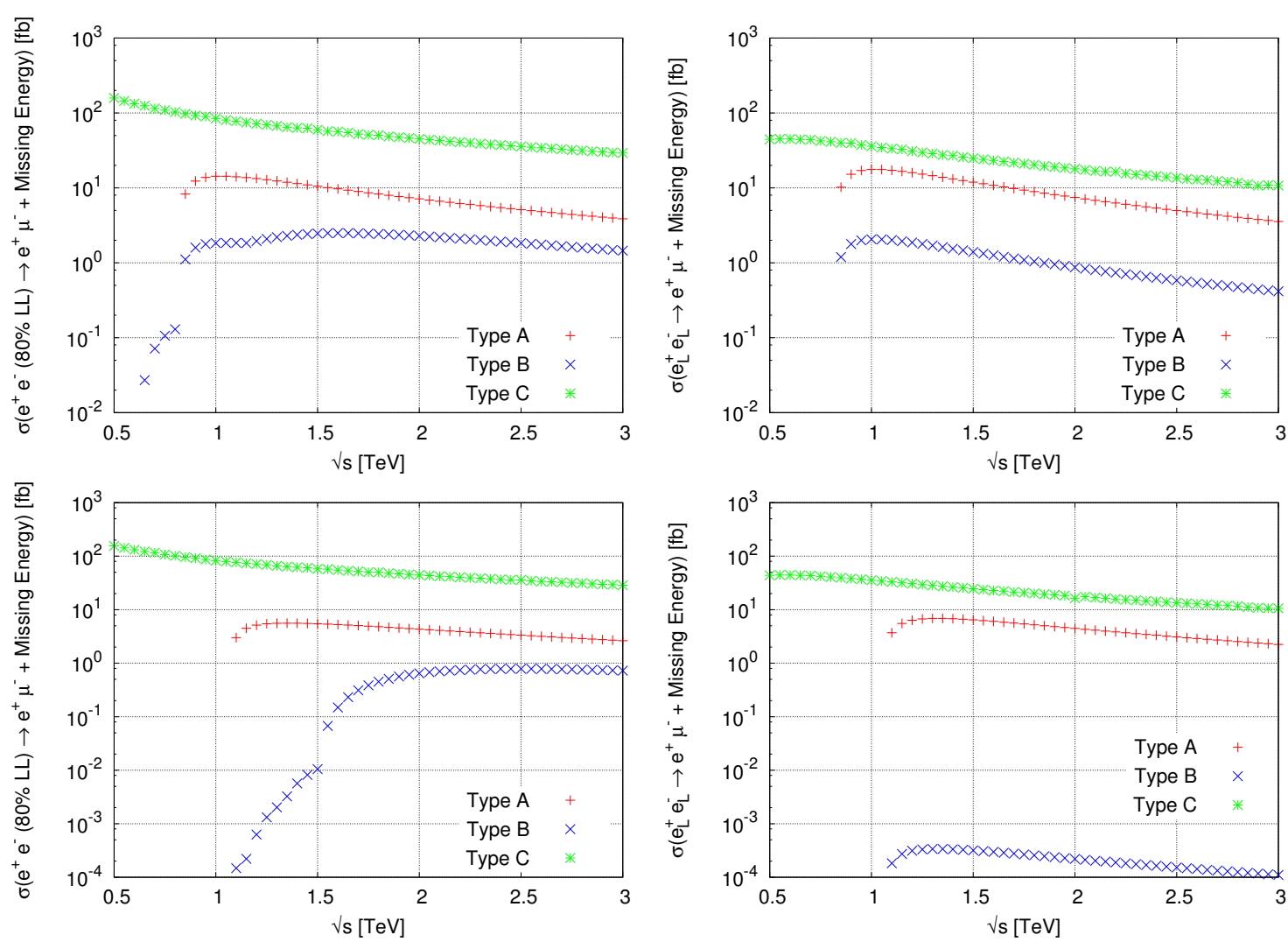

Figure 7. Cross section for $e^{+} e^{-} \rightarrow e^{+} \mu^{-}+E_{\text {miss }}^{T}$ (with $\left.E_{\text {miss }}^{T}=2 \chi_{1}^{0}, 2 \chi_{1}^{0}+(2,4) \nu,(2,4) \nu\right)$, for points C-light and C-heavy (upper and lower panels, respectively), as a function of the centre of mass energy, $\sqrt{s}$, for polarised beams. Left panels: $\left(P_{e^{+}}, P_{e^{-}}\right)=(-80 \%,-80 \%)$; right panels $\left(P_{e^{+}}, P_{e^{-}}\right)=(-100 \%,-100 \%)$. We fix $M_{R}=10^{12} \mathrm{GeV}$, and again denote the signal (A) with red crosses, the SUSY charged current background (B) with blue times, and the SM charged current background $(\mathrm{C})$ by green asterisks. In both cases we have taken a degenerate right-handed neutrino spectrum, and set $\theta_{13}=10^{\circ}$.

tain a signal of cLFV, as possibly induced by a SUSY seesaw. Regarding the expected number of events in the polarised cases, for $\sqrt{s}=2 \mathrm{TeV}$, and $\mathcal{L}=0.5(3) \mathrm{ab}^{-1}$, one expects (in both $100 \%$ and $80 \%$ polarization cases), values of order of $5 \times 10^{3}\left(3 \times 10^{4}\right)$ for C-light and $10^{3}\left(10^{4}\right)$ for point C-heavy.

The results displayed in figure 7 correspond to a fixed value of the (degenerate) righthanded neutrino scale $\left(M_{R}=10^{12} \mathrm{GeV}\right)$. Conducting an analysis similar to that leading to the right-handside panels of figures 4 and 5 (i.e., study of $e^{+} e^{-} \rightarrow e^{+} \mu^{-}+E_{\text {miss }}^{T}$ as a function of $M_{R}$ ), we have verified that for sufficiently large values of $M_{R}$, which are still compatible with the bounds from $\operatorname{BR}(\mu \rightarrow e \gamma)$, the signal cross section is larger than that of the SUSY background; in particular, this occurs for spectra similar to that of point C-heavy. If, as discussed above, dedicated cuts allow to reduce the SM backgrounds, then one could also infer some information concerning the scale of a possible underlying type I seesaw.

Again, we complete this analysis by displaying in figure 8 the significance of the signal for both points $\mathrm{C}$ in the case of an $80 \%$ and in the "ideal" $100 \%$ case beam polarisation. 

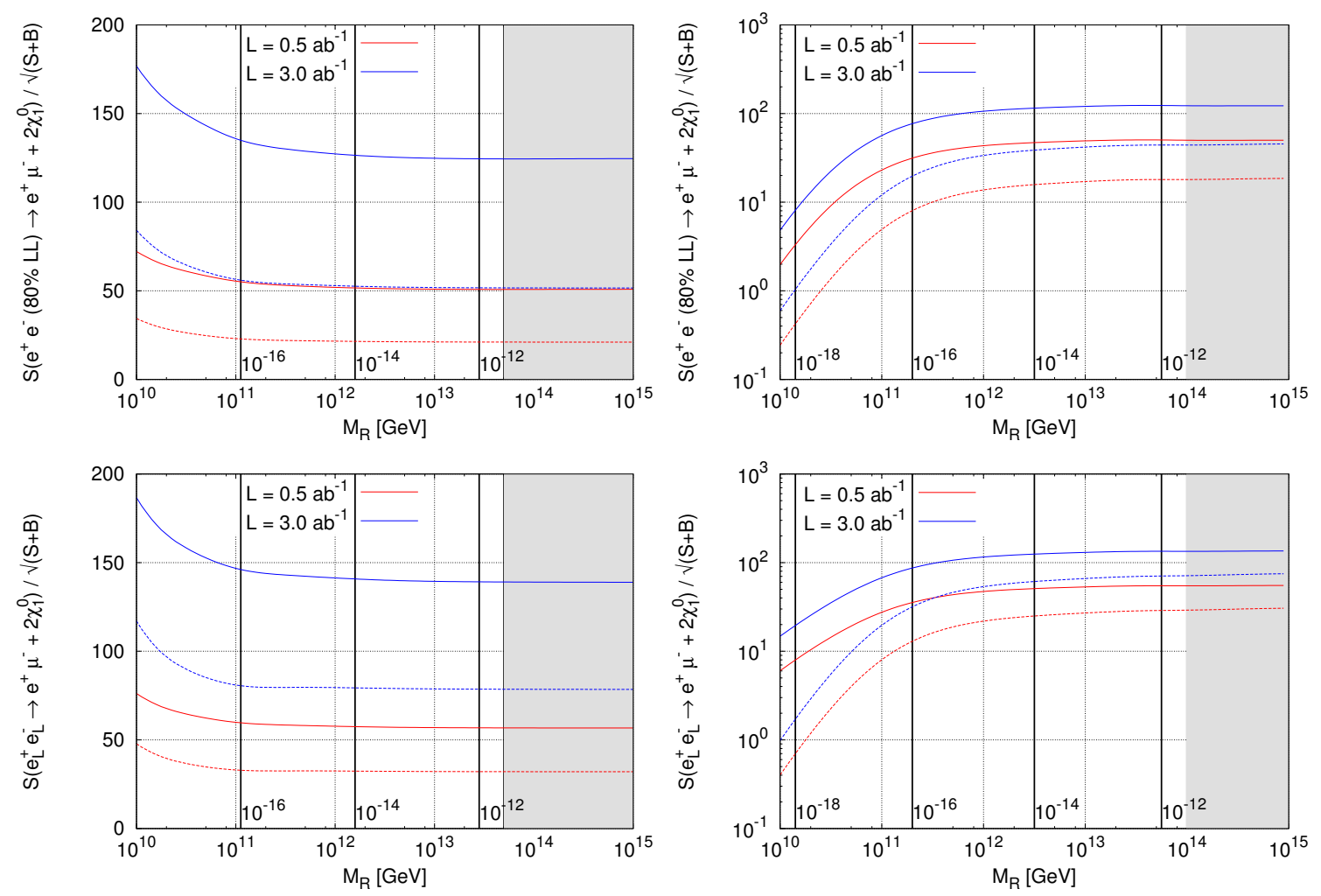

Figure 8. Significance of the signal for points C-light (left) and C-heavy (right), as a function of the seesaw scale $\left(M_{R}\right)$, for $\sqrt{s}=2 \mathrm{TeV}$, with $80 \%$ (upper) and $100 \%$ (lower panels), beam polarisation. Line and colour code as in figure 6 .

The results thus obtained further suggest that cLFV will be indeed observable at a Linear Collider in $e^{+} e^{-}$collisions: under an $80 \%$ positron and electron beam polarisation, the significances are typically above 10 , and can be even close to 100 .

\section{$5.2 e^{-} e^{-}$beam option}

We now proceed to explore the very interesting LC feature of $e^{-} e^{-}$collisions. In figure 9 , we display the cross section for $e^{-} e^{-} \rightarrow e^{-} \mu^{-}+E_{\text {miss }}^{T}$ (with $E_{\text {miss }}^{T}=2 \chi_{1}^{0}, 2 \chi_{1}^{0}+(2,4) \nu,(2,4) \nu$ ), for points C-light and C-heavy (upper and lower panels, respectively), as a function of the centre of mass energy, $\sqrt{s}$, and of the seesaw scale $M_{R}$.

Although the cross sections for $e^{-} e^{-} \rightarrow W^{-} W^{-}$production are extremely tiny, the SM does still contribute with a dominant background due to $e^{-} e^{-} \rightarrow e^{-} \nu W^{-}$processes (notice that the $\sigma_{\text {prod }}\left(e^{-} e^{-} \rightarrow\left(\mu^{-}, \tau^{-}\right) W^{-} \nu\right) \simeq 0$ ). As discussed in section 4 , processes contributing to the non- $\tau$ SUSY background are only present if the spectrum is such that $m_{\tilde{\ell}} \gtrsim m_{\chi_{1}^{ \pm}}$, which is not the case for points C. Thus, and as argued in the case of $e^{+} e^{-}$ beams, for SUSY spectra along the co-annihilation region, and should appropriate cuts allow to reduce the SM $W$-background, the prospects of disentangling the cLFV effects of a potential type I SUSY seesaw at a Linear Collider are very promising for the $e^{-} e^{-}$ beam option. As previously discussed in section 5.1, due to the nature of the dominant SM 

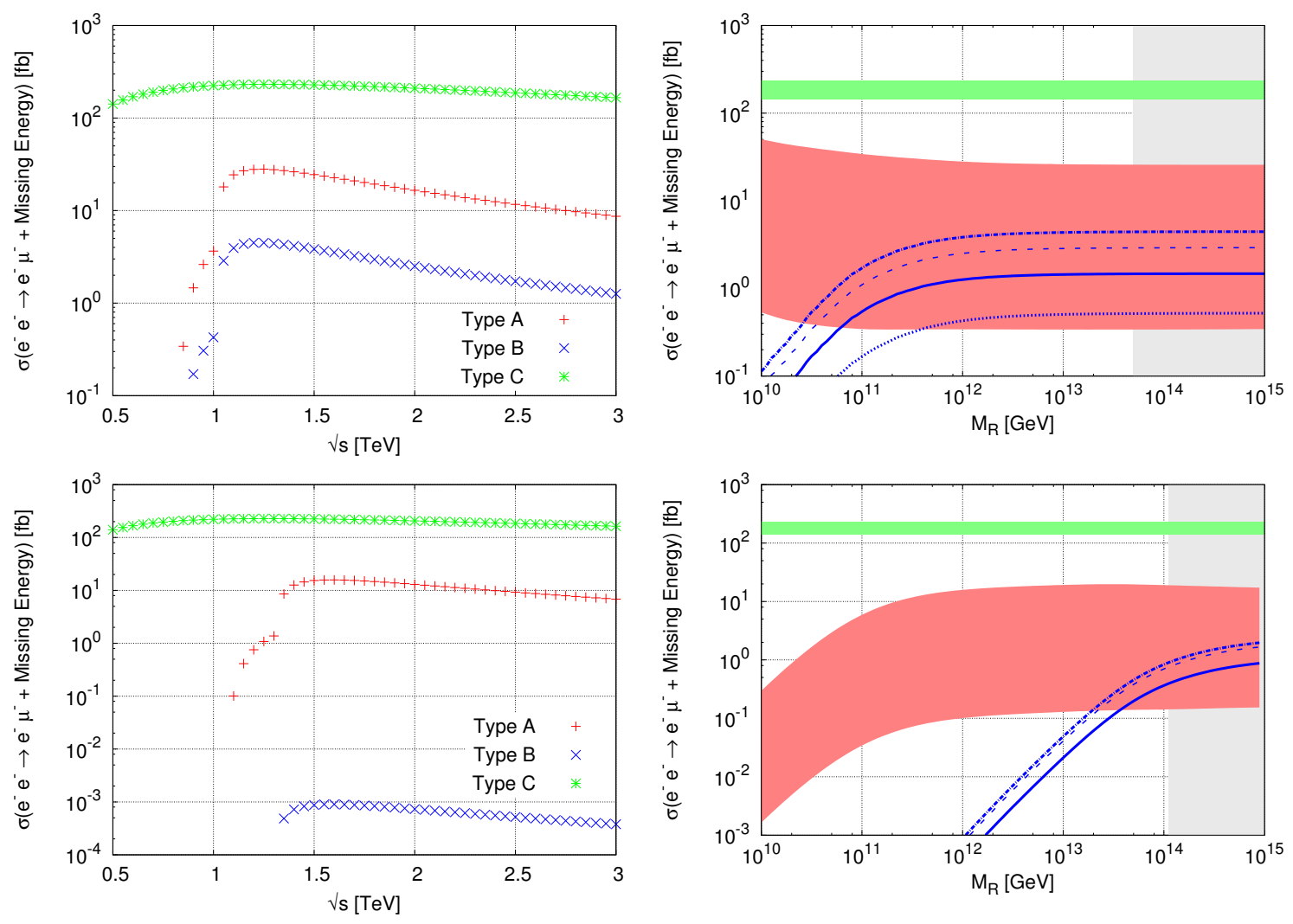

Figure 9. On the left, cross section for $e^{-} e^{-} \rightarrow e^{-} \mu^{-}+E_{\text {miss }}^{T}$ (with $E_{\text {miss }}^{T}=2 \chi_{1}^{0}, 2 \chi_{1}^{0}+$ $(2,4) \nu,(2,4) \nu)$, for points C-light and C-heavy (upper and lower panels, respectively), as a function of the centre of mass energy, $\sqrt{s}$. On the right, cross section for $e^{-} e^{-} \rightarrow e^{-} \mu^{-}+E_{\text {miss }}^{T}$ (with $\left.E_{\text {miss }}^{T}=2 \chi_{1}^{0}, 2 \chi_{1}^{0}+(2,4) \nu,(2,4) \nu\right)$, for points C-light and C-heavy (upper and lower panels, respectively), as a function of the right-handed neutrino mass $\left(M_{R}\right)$. In both cases we have taken a degenerate right-handed neutrino spectrum, and set $\theta_{13}=10^{\circ}$. Line and colour code as in figure 4 .

background, polarising the beams only translates into a small increase of the total (signal) cross section. This is illustrated in figure 10 .

Still, notice that the effect of the LL polarisation is clearly visible in the disappearance of the SUSY (signal and background) processes occurring for the lower values of $\sqrt{s}$ : as can be inferred from the associated spectrum shown in table 2 , these would correspond to the production of (at least) one right-handed slepton. For a c.o.m. energy of $2 \mathrm{TeV}$, the expected number of events for C-light is around $3 \times 10^{4}\left(2 \times 10^{5}\right)$ for $\mathcal{L}=0.5(3) \mathrm{ab}^{-1}$; in the case of C-heavy the expected number of events varies between 300 and $2 \times 10^{4}$, for $\mathcal{L}=0.5 \mathrm{ab}^{-1}$, and between 2000 and $10^{5}$ for $\mathcal{L}=3 \mathrm{ab}^{-1}$.

In order to fully explore the potential of the results so far obtained, let us assume that SUSY has been discovered, with a spectrum resembling one of the points in table 2, and that a type I seesaw is indeed the unique source of LFV at work. We focus on points C-light and C-heavy, as these are associated with a potentially large number of events, as can be seen in figure 11 . 

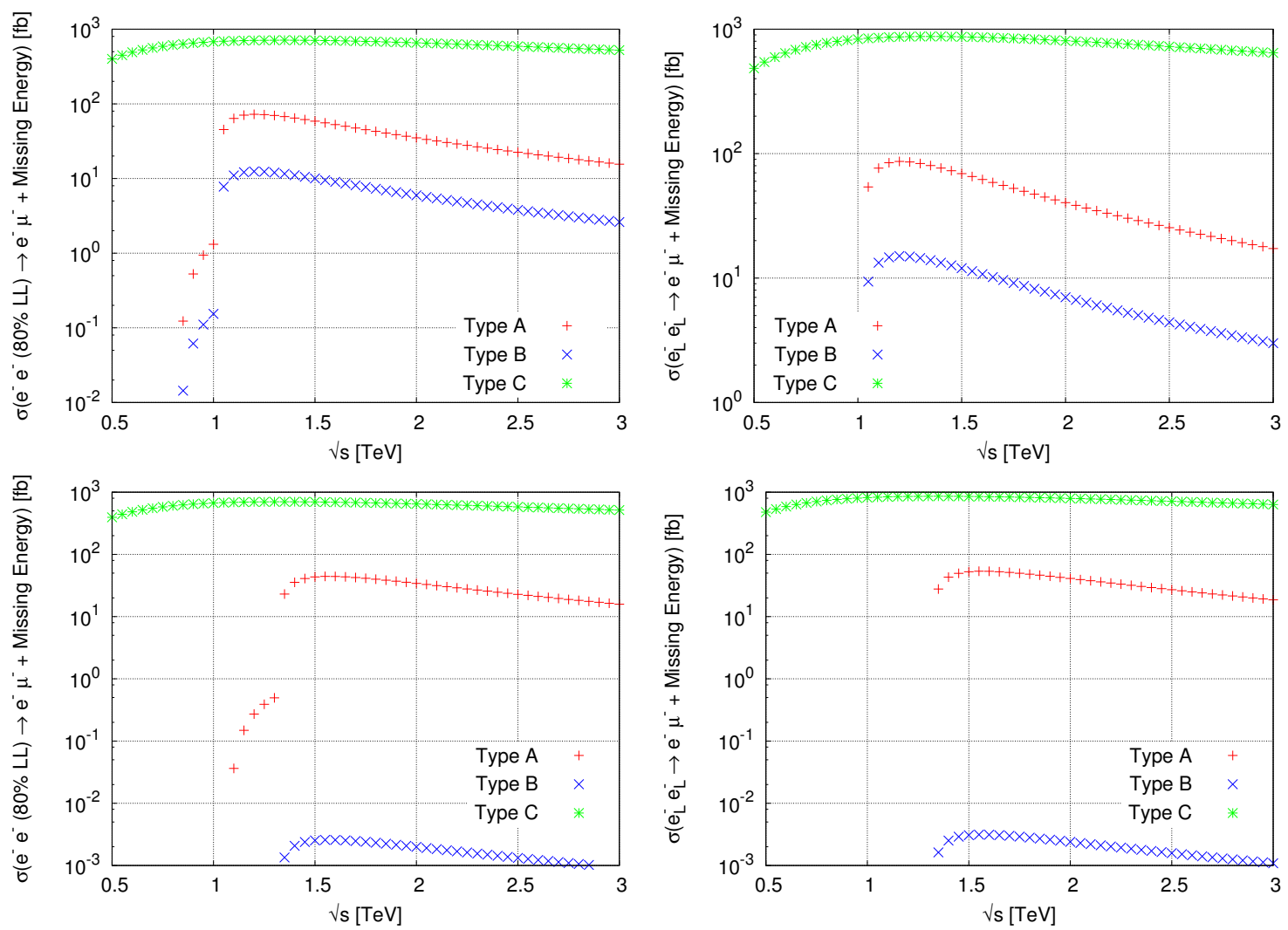

Figure 10. Cross section for $e^{-} e^{-} \rightarrow e^{-} \mu^{-}+E_{\text {miss }}^{T}$ (with $\left.E_{\text {miss }}^{T}=2 \chi_{1}^{0}, 2 \chi_{1}^{0}+(2,4) \nu,(2,4) \nu\right)$, for points C-light and C-heavy (upper and lower panels, respectively), as a function of the centre of mass energy, $\sqrt{s}$, for polarised beams. On the left: $\left(P_{e^{-}}, P_{e^{-}}\right)=(-80 \%,-80 \%)$; on the right: $\left(P_{e^{-}}, P_{e^{-}}\right)=(-100 \%,-100 \%)$. We have taken a degenerate right-handed neutrino spectrum $\left(M_{R}=10^{12} \mathrm{GeV}\right)$ with $\theta_{13}=10^{\circ}$.

To illustrate our discussion, we consider the expected number of events $e^{-} e^{-} \rightarrow e^{-} \mu^{-}+$ $E_{\text {miss }}^{T}$ (with $E_{\text {miss }}^{T}=2 \chi_{1}^{0}$ ), in the case of a realistic scenario for the beam polarisation, i.e. $\left(P_{e^{-}}, P_{e^{-}}\right)=(-80 \%,-80 \%)$. We further assume that dedicated cuts will have allowed to significantly reduce the ( $W$-strahlung) SM background. Notice that it is quite likely that the cuts needed to reduce the background will also somewhat reduce the signal; this would naturally imply a rescaling of the corresponding curves of figure 11.

Firstly, if at the LC, no cLFV event of the type $e^{-} e^{-} \rightarrow e^{-} \mu^{-}+E_{\text {miss }}^{T}$ is observed (for any possible luminosity and c.o.m. energy), then a high-scale type I SUSY seesaw should be clearly disfavoured as an hypothesis for the (unique) underlying source of LFV. Secondly, let us assume that a sizable number of events is indeed collected: if such a number can be accommodated by the predictions (as illustrated by the curves of figure 11), then one can derive information about the seesaw scale. For instance, if for a C-heavy-like spectrum, more than $10^{5}$ events are observed (for $\mathcal{L} \sim 3 \mathrm{ab}^{-1}$ ), then the seesaw scale $\left(M_{R}\right.$, or in the hierarchical case, $M_{N_{3}}$ ) should be above $10^{12} \mathrm{GeV}$. Furthermore, compatibility with $\mathrm{BR}(\mu \rightarrow e \gamma)$ bounds also puts an upper bound on this scale, so that in this case one 

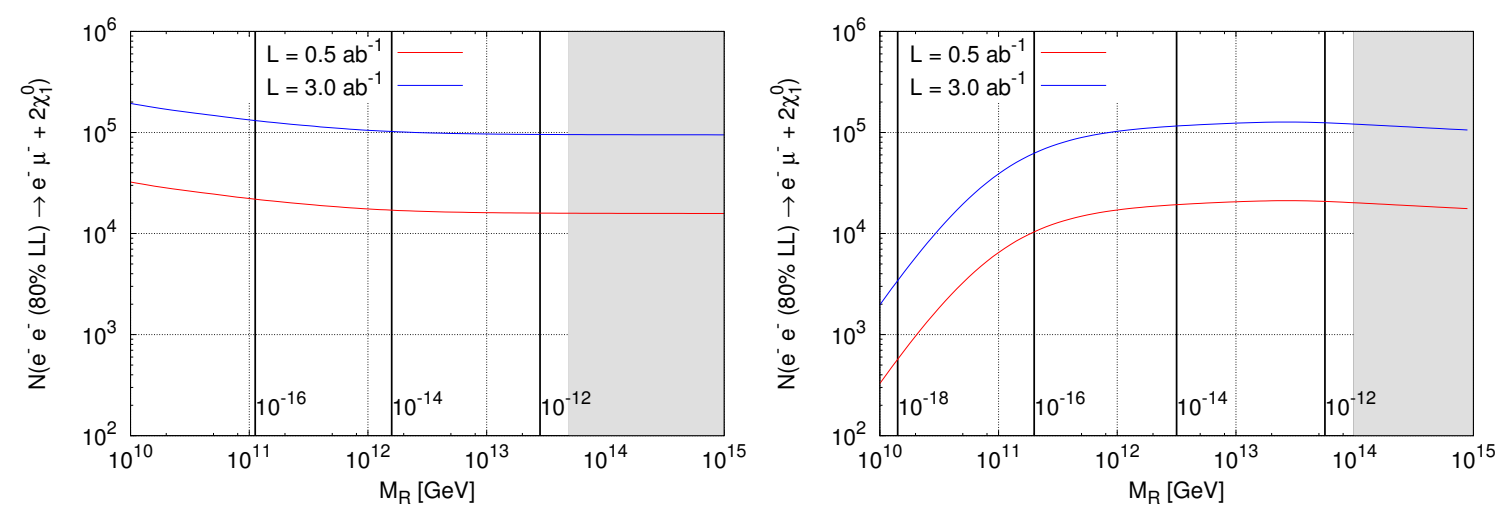

Figure 11. Number of events for $e^{-} e^{-} \rightarrow e^{-} \mu^{-}+E_{\text {miss }}^{T}$ (with $E_{\text {miss }}^{T}=2 \chi_{1}^{0}$ ), for points C-light (left) and C-heavy (right), as a function of the seesaw scale $M_{R}$, for $\left(P_{e^{-}}, P_{e^{-}}\right)=(-80 \%,-80 \%)$ polarised beams. In both cases, we fix $\sqrt{s}=2 \mathrm{TeV}$, and we have taken a degenerate right-handed neutrino spectrum, with $\theta_{13}=10^{\circ}$. Vertical lines denote the $M_{R}$-corresponding value of $\operatorname{BR}(\mu \rightarrow e \gamma)$ while the (grey) shaded region represents values of $M_{R}$ already excluded by the present experimental bound on $\operatorname{BR}(\mu \rightarrow e \gamma)$.

would be led to $10^{12} \mathrm{GeV} \lesssim M_{R} \lesssim 10^{14} \mathrm{GeV}$. The observation of a $\mu \rightarrow e \gamma$ decay at MEG can be instrumental: let us assume that a $\operatorname{BR}(\mu \rightarrow e \gamma) \sim \mathcal{O}\left(10^{-12}\right)$ is indeed found. In this case, any number of $e^{-} e^{-} \rightarrow e^{-} \mu^{-}+E_{\text {miss }}^{T}$ events below $10^{5}$ renders the two cLFV manifestations incompatible with the assumption of a unique source of LFV: either some (unaccounted for) destructive interference occurred in the high-energy processes, which lowered the number of events, or then there are additional sources of LFV, only manifest in the low-energy observables, which account for the enhancement of the $\operatorname{BR}(\mu \rightarrow e \gamma)$. However, if the number of events corroborates the theoretical expectations for a seesaw scale that would indeed account for such a $\operatorname{BR}(\mu \rightarrow e \gamma)$, then this interplay between highand low-energy observables strengthens the hypothesis of a type I seesaw as the unique source of LFV.

Should there be additional data on cLFV from the LHC (for example, in association with neutralino decays into sleptons), then one can fully explore the synergy between a large array of high- and low-energy cLFV observables.

Finally, we present in figure 12, the expected significance of the signal, for unpolarised, $80 \%$ polarised and fully polarised electron beams. As in the case of $e^{+} e^{-} \rightarrow e^{+} \mu^{-}+E_{\text {miss }}^{T}$ collisions, an $80 \%$ polarised electron beam configuration would already allow to have a significance of the signal around 10, for both points C-light and C-heavy, even for an integrated luminosity of $0.5 \mathrm{ab}^{-1}$ (in the case of C-heavy, only for $M_{R} \gtrsim 10^{11} \mathrm{GeV}$ ). We again stress that dedicated cuts could even further improve these values.

\section{3 cLFV from $\mu^{-} \mu^{-}$final states}

To complete our discussion, we finally comment on what might possibly be a "golden channel" for the detection of cLFV at a Linear Collider.

$e^{-} e^{-} \rightarrow \mu^{-} \mu^{-}+E_{\text {miss }}^{T}$ events turn out to be extremely clean probes of cLFV, from an experimental and a theoretical point of view: firstly, the efficiency of the muon detectors can 

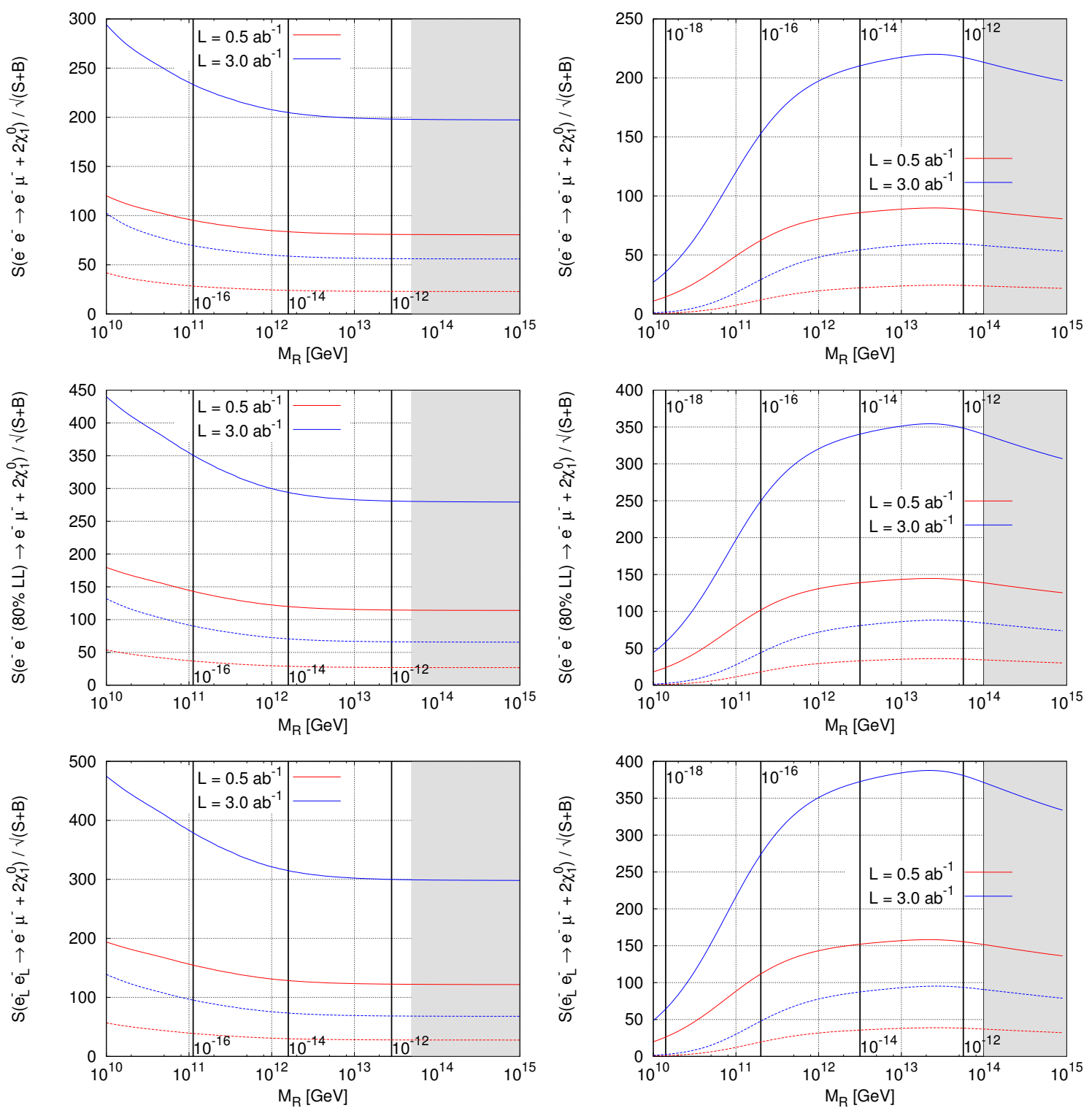

Figure 12. Significance of the signal $e^{-} e^{-} \rightarrow e^{-} \mu^{-}+E_{\text {miss }}^{T}$ for points C-light (left) and C-heavy (right), as a function of the seesaw scale $\left(M_{R}\right)$, for $\sqrt{s}=2 \mathrm{TeV}$, and for unpolarised (upper), $80 \%$ (mid), and fully polarised (lower panels) $e^{-}$beams. Line and colour code as in figure 6 .

be fully explored when looking for $e^{-} e^{-} \rightarrow \mu^{-} \mu^{-}+E_{\text {miss }}^{T}$ signals; secondly, and especially when compared to the other (already discussed) signals - i.e. $e^{+(-)} e^{-} \rightarrow e^{+(-)} \mu^{-}+E_{\text {miss }}^{T}$ - the SM model background is extremely tiny in this case. SUSY background processes are still present, but are subdominant when compared to the signal, as the corresponding cross sections differ by around one (four) order of magnitude for C-light (C-heavy), as is illustrated on figure 13.

For the case of fully polarised electron beams, and a c.o.m. energy of $2 \mathrm{TeV}$, the expected number of events for C-light is $\sim 1000$ (6000) for $\mathcal{L}=0.5(3) \mathrm{ab}^{-1}$; in the case 

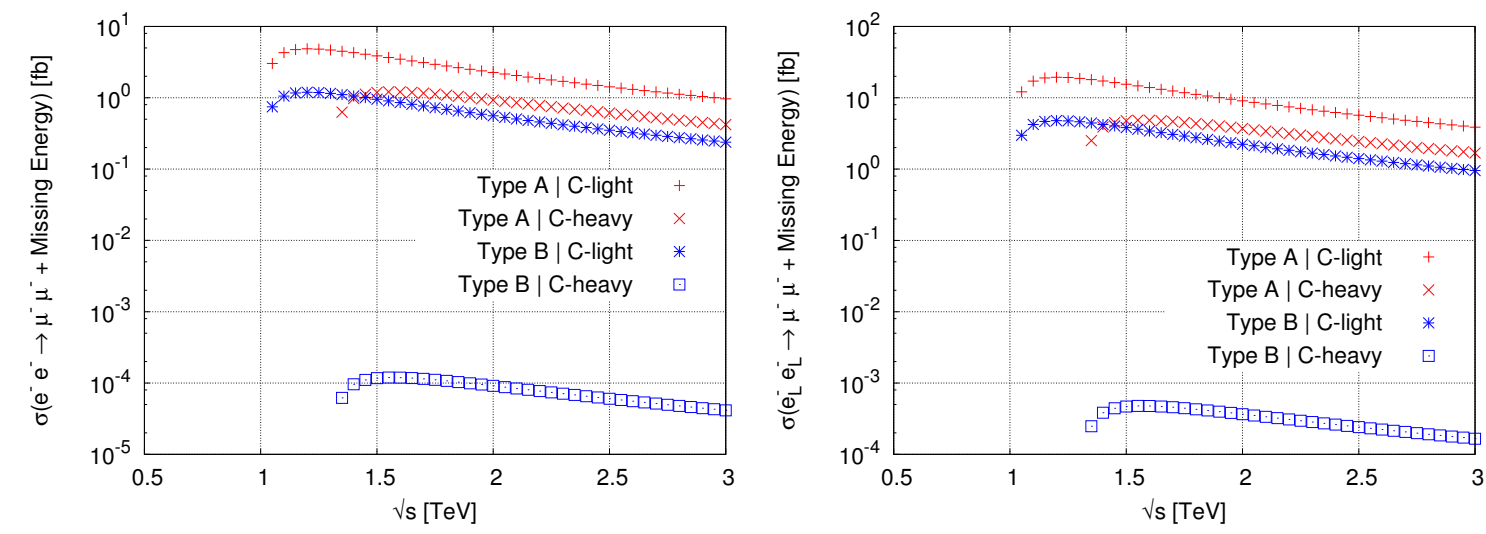

Figure 13. Cross section for $e^{-} e^{-} \rightarrow \mu^{-} \mu^{-}+E_{\text {miss }}^{T}$ (with $E_{\text {miss }}^{T}=2 \chi_{1}^{0}, 2 \chi_{1}^{0}+(2,4) \nu,(2,4) \nu$ ), for points C-light and C-heavy as a function of the centre of mass energy, $\sqrt{s}$, in the unpolarised beam case (left) and for fully polarised beams $\left(P_{e^{-}}, P_{e^{-}}\right)=(-100 \%,-100 \%)$ (right). C-light: the signal (SUSY background) is denoted by red crosses (blue asterisks); C-heavy: the signal (SUSY background) is represented by red times (blue squares). We have taken a degenerate right-handed neutrino spectrum $\left(M_{R}=10^{12} \mathrm{GeV}\right)$ and set $\theta_{13}=10^{\circ}$.

of C-heavy the expected number of events is $\sim 500$ (3000). Should this be the case, one would clearly identify the presence of a new physics scenario, such as the SUSY seesaw - always under the assumption of having a unique source of Lepton Flavour Violation present. Naturally, one would be also confirming the Majorana nature of the exchanged particles in the $t$-channel.

\section{Concluding remarks}

Following recent developments in lepton physics and the new physics searches at the LHC, we have revisited the potential of a Linear Collider concerning the study of lepton flavour violation: in particular, we investigated cLFV in $e^{+} e^{-}$and $e^{-} e^{-}$collisions in the framework of a type I SUSY seesaw. Due to the leptonic mixing associated with charged current interactions $\left(U_{\mathrm{MNS}}\right)$, cLFV will always occur in any model that can accommodate neutrino oscillations, independently from any mechanism of mass generation or new physics model.

If it is to explain neutrino masses and mixings with natural values for the Yukawa couplings, the type I seesaw is impossible to probe directly (at high scale); however, and when embedded into supersymmetric theories, it might radiatively induce several cLFV phenomena, which can be manifest in both low-energy observables and in a number of processes at high-energy colliders.

In this work, and following early analyses [78, 79], we have considered $e^{ \pm} e^{-} \rightarrow \ell_{i}^{ \pm} \ell_{j}^{-}+$ $E_{T}^{\text {miss }}$ and discussed how the signatures of a type I SUSY seesaw can be disentangled from the SM and/or the MSSM, when the latter are effectively enlarged to accommodate low-energy neutrino data (i.e. $U_{\mathrm{MNS}} \neq 1$ ). To fully explore the LC beam potentialities and maximise the existent synergie with very sensitive low-energy observables such as $\mu \rightarrow e \gamma$ decay and $\mu-e$ conversion in nuclei, we selected as "signal" the processes $e^{ \pm} e^{-} \rightarrow$ 
$e^{ \pm} \mu^{-}+2 \chi_{1}^{0}$. We considered the possibility of electron and positron beam polarisation, investigating the prospects for c.o.m. energies in the interval $[500 \mathrm{GeV}, 3 \mathrm{TeV}]$ and for nominal integrated luminosities of $\mathcal{L}=0.5$ and $3 \mathrm{ab}^{-1}$.

Although the SM background typically dominates, we have shown that for an $e^{+} e^{-}$ Linear Collider, running for instance at $\sqrt{s}=2 \mathrm{TeV}$, and prior to any selection cuts, $80 \%$ polarised beams would provide as much as $\sim 10^{3}\left(10^{4}\right) e^{+} e^{-} \rightarrow e^{+} \mu^{-}+2 \chi_{1}^{0}$ events for the above values of $\mathcal{L}$ (in the case of a relatively light SUSY spectrum, and in the low $\tan \beta$ regime).

Furthermore the $e^{-} e^{-}$beam option provides one of the cleanest experimental setups to probe not only lepton number violation but also lepton flavour violation. Even without beam polarisation, $e^{-} e^{-}$collisions offer excellent prospects for disentangling the specific cLFV effects of a type-I SUSY seesaw, in particular for SUSY spectra along the so-called co-annihilation region. For instance, for $\sqrt{s}=2 \mathrm{TeV}$, one could expect as much as $\sim 10^{4}$ $\left(10^{5}\right) e^{-} e^{-} \rightarrow e^{-} \mu^{-}+2 \chi_{1}^{0}$ events for an integrated luminosity of $\mathcal{L}=0.5(3) \mathrm{ab}^{-1}$.

In view of these promising prospects, the confrontation of the theoretical estimations for a given reconstructed SUSY spectrum with future data from dedicated lepton flavour violating studies at a LC would allow to derive information about the scale of the underlying neutrino mass generation mechanism. Moreover, in the framework of a type I SUSY seesaw, there would be a strict correlation between the processes $e^{-} e^{-} \rightarrow e^{-} \mu^{-}+2 \chi_{1}^{0}$ and lowenergy observables such as BR $(\mu \rightarrow e \gamma)$. As discussed in this paper, the interplay of $\mathrm{FV}$ at a LC (especially for the $e^{-} e^{-}$beam option) and a signal at MEG would strongly substantiate the hypothesis of a type-I SUSY seesaw.

The present study only addressed cLFV at a Linear Collider from a phenomenological point of view. The results obtained here (signal and background cross sections, expected number of events and associated statistical significance) are just a first step of a larger study: the analysis can and should be improved by a full fledged simulation, generating the events, applying kinematic cuts (see examples above), as well as acceptance cuts of a generic detector.

\section{Acknowledgments}

We are indebted to O. Panella for many valuable exchanges and suggestions, and for a critical reading of the manuscript. A.M.T. is grateful to M. Battaglia for interesting discussions, and input on LC benchmarks. This work has been done partly under the ANR project CPV-LFV-LHC NT09-508531. The work of A.J.R.F. has been supported by Fundação para a Ciência e a Tecnologia through the fellowship SFRH/BD/64666/2009. A.A., A.J.R.F. and A.M.T. acknowledge partial support from the European Union FP7 ITN INVISIBLES (Marie Curie Actions, PITN-GA-2011-289442). A.J.R.F. and J.C.R. also acknowledge the financial support from the EU Network grant UNILHC PITN-GA2009-237920 and from Fundação para a Ciência e a Tecnologia grants CFTP-FCT UNIT 777, CERN/FP/116328/2010 and PTDC/FIS/102120/2008. 


\section{A Particle production and decay}

In our numerical analysis we have considered all relevant contributions to

$$
e^{ \pm} e^{-} \rightarrow X \rightarrow\{Y\} \rightarrow e^{ \pm} \mu^{-}+(0,2,4) \chi_{1}^{0}+(1,2,3) \bar{\nu} \nu
$$

with final state particle number no greater than 8 . In the above, $X$ denotes any element of the set displayed in eq. (A.2), and $\{Y\}$ the possible two-body decays, as listed below, in eqs. (A.3)-(A.9). The primary production modes (on-shell) are as follows: ${ }^{6}$

$$
e^{+} e^{-} \rightarrow\left\{\begin{array} { l } 
{ \chi _ { A } ^ { 0 } \chi _ { B } ^ { 0 } } \\
{ \chi _ { A } ^ { \pm } \chi _ { B } ^ { \mp } } \\
{ \tilde { \ell } _ { i } ^ { + } \tilde { \ell } _ { j } ^ { - } } \\
{ \tilde { \nu } _ { i } \tilde { \nu } _ { j } ^ { * } } \\
{ h Z , H A , H ^ { + } H ^ { - } } \\
{ W ^ { + } W ^ { - } } \\
{ \tau ^ { + } \tau ^ { - } } \\
{ W ^ { + } \ell _ { i } ^ { - } \overline { \nu } + \text { c.c. } }
\end{array} e ^ { - } e ^ { - } \rightarrow \left\{\begin{array}{l}
\tilde{\ell}_{i}^{-} \tilde{\ell}_{j}^{-} \\
W^{-} \ell_{i}^{-} \nu
\end{array} .\right.\right.
$$

In view of the final states under study, the above produced states were allowed to decay, leading to a two-body decay cascade; among the final states will be those of the type (A), (B) and (C) processes (see eqs. (3.2), (4.1)), corresponding to the cLFV signal and to the SUSY and SM backgrounds. The following two-body decays (plus the 3-body $\tau$ decays) were considered, obeying kinematical and simple selection rules (denoted by superscripts in the decay products). The former are a natural consequence of the hierarchy of the different considered spectra ${ }^{7}$

$$
\begin{aligned}
& \tilde{e}_{L}^{-} \rightarrow\left\{\begin{array}{l}
\left(\chi_{1}^{0}, \chi_{2}^{0}\right) \ell_{i}^{-} \\
h \tilde{\tau}_{1}^{-} \\
Z\left(\tilde{\tau}_{2}^{-}, \tilde{\tau}_{1}^{-}\right) \\
\left(\chi_{1}^{-}, \chi_{2}^{-}\right) \nu^{(1)} \\
W^{-} \tilde{\nu}_{\tau}
\end{array}\right. \\
& \tilde{\mu}_{L}^{-} \rightarrow\left\{\begin{array}{l}
\left(\chi_{1}^{0}, \chi_{2}^{0}\right) \ell_{i}^{-} \\
h \tilde{\tau}_{1}^{-} \\
Z\left(\tilde{\tau}_{2}^{-}, \tilde{\tau}_{1}^{-}\right) \\
\left(\chi_{1}^{-}, \chi_{2}^{-}\right) \nu^{(1)} \\
W^{-} \tilde{\nu}_{\tau}
\end{array}\right. \\
& \tilde{\tau}_{2}^{-} \rightarrow\left\{\begin{array}{l}
\left(\chi_{1}^{0}, \chi_{2}^{0}\right) \ell_{i}^{-} \\
h \tilde{\tau}_{1}^{-} \\
Z\left(\tilde{e}_{L}^{-}, \tilde{\mu}_{L}^{-}, \tilde{\tau}_{1}^{-}\right) \\
\left(\chi_{1}^{-}, \chi_{2}^{-}\right) \nu^{(1)}
\end{array}\right. \\
& \tilde{e}_{R}^{-} \rightarrow\left\{\begin{array}{l}
\left(\chi_{1}^{0}, \chi_{2}^{0}\right) \ell_{i}^{-} \\
\left(\chi_{1}^{-}, \chi_{2}^{-}\right) \nu^{(1)}
\end{array}\right. \\
& \tilde{\mu}_{R}^{-} \rightarrow\left\{\begin{array}{l}
\left(\chi_{1}^{0}, \chi_{2}^{0}\right) \ell_{i}^{-} \\
\left(\chi_{1}^{-}, \chi_{2}^{-}\right) \nu^{(1)}
\end{array}\right. \\
& \tilde{\tau}_{1}^{-} \rightarrow\left\{\begin{array}{l}
\left(\chi_{1}^{0}, \chi_{2}^{0}\right) \ell_{i}^{-} \\
\left(\chi_{1}^{-}, \chi_{2}^{-}\right) \nu^{(1)}
\end{array}\right. \\
& \tilde{\nu}_{e} \rightarrow\left\{\begin{array}{l}
W^{+}\left(\tilde{\tau}_{2}^{-}, \tilde{e}_{R}^{-}, \tilde{\mu}_{R}^{-}, \tilde{\tau}_{1}^{-}\right) \\
\left(\chi_{1}^{0}, \chi_{2}^{0}\right) \nu \\
\left(\chi_{1}^{+}, \chi_{2}^{+}\right) \ell_{i}^{-}(1)
\end{array}\right. \\
& \tilde{\nu}_{\mu} \rightarrow\left\{\begin{array}{l}
W^{+}\left(\tilde{\tau}_{2}^{-}, \tilde{e}_{R}^{-}, \tilde{\mu}_{R}^{-}, \tilde{\tau}_{1}^{-}\right) \\
\left(\chi_{1}^{0}, \chi_{2}^{0}\right) \nu \\
\left(\chi_{1}^{+}, \chi_{2}^{+}\right) \ell_{i}^{-(1)}
\end{array}\right. \\
& \tilde{\nu}_{\tau} \rightarrow\left\{\begin{array}{l}
W^{+}\left(\tilde{e}_{R}^{-}, \tilde{\mu}_{R}^{-}, \tilde{\tau}_{1}^{-}\right) \\
\left(\chi_{1}^{0}, \chi_{2}^{0}\right) \nu \\
\left(\chi_{1}^{+}, \chi_{2}^{+}\right) \ell_{i}^{-}{ }^{(1)}
\end{array}\right.
\end{aligned}
$$

\footnotetext{
${ }^{6}$ Notice that we have excluded $e^{+} e^{-} \rightarrow H Z, e^{+} e^{-} \rightarrow h A$ and $e^{-} e^{-} \rightarrow W^{-} W^{-}$since, throughout the parameter space explored, we have found the associated cross sections to be highly suppressed: $\sigma_{H Z}<$ $1.1 \times 10^{-4} \mathrm{fb}, \sigma_{h A}<2 \times 10^{-5} \mathrm{fb}$ and $\sigma_{W^{-} W^{-}}<1.1 \times 10^{-21} \mathrm{fb}$.

${ }^{7}$ For cases of nearly-degenerate LSP and NLSP, typically associated to the dark matter co-annihilation region, the NLSP (the lightest stau) becomes extremely long-lived, potentially decaying outside the detector. This is the case of point C-heavy, for which the $\tilde{\tau}_{1}$ 's lifetime is $\mathcal{O}(100 \mu \mathrm{s})$.
} 


$$
\begin{aligned}
& \chi_{1}^{+} \rightarrow\left\{\begin{array}{l}
W^{+} \chi_{1}^{0} \\
\tilde{\ell}_{i}^{+} \nu(2) \\
\tilde{\nu}_{i} \ell_{j}^{+}(3)
\end{array}\right. \chi_{2}^{+} \rightarrow\left\{\begin{array}{l}
W^{+}\left(\chi_{1}^{0}, \chi_{2}^{0}\right) \\
(Z, h) \chi_{1}^{+} \\
\tilde{\ell}_{i}^{+} \nu(2) \\
\tilde{\nu}_{i} \ell_{j}^{+}(3)
\end{array}\right. \\
& \chi_{2}^{0} \rightarrow\left\{\begin{array}{l}
\tilde{\nu}_{i} \bar{\nu}+\text { c.c. }{ }^{(3)} \\
(Z, h) \chi_{1}^{0} \\
\tilde{\ell}_{i}^{+} \ell_{j}^{-}+\text {c.c. }{ }^{\left(2^{*}\right)}
\end{array}\right. \\
& \tau_{3}^{ \pm} \rightarrow\left\{\begin{array}{l}
(Z, h)\left(\chi_{1}^{0}, \chi_{2}^{0}\right) \\
\chi_{1}^{+} W^{-}+\text {c.c. }
\end{array}\right.
\end{aligned}
$$

$$
H \rightarrow\left\{\begin{array}{l}
\tilde{e}_{L}^{+} \tilde{e}_{L}^{-} \\
\tilde{e}_{R}^{+} \tilde{e}_{R}^{-} \\
\tilde{\mu}_{L}^{+} \tilde{\mu}_{L}^{-} \\
\tilde{\mu}_{L}^{+}\left(\tilde{\tau}_{2}^{-}, \tilde{\mu}_{R}^{-}, \tilde{\tau}_{1}^{-}\right)+\text {c.c. } \\
\tilde{\tau}_{2}^{+} \tilde{\tau}_{2}^{-} \\
\tilde{\tau}_{2}^{+}\left(\tilde{\mu}_{R}^{-}, \tilde{\tau}_{1}^{-}\right)+\text {c.c. } \\
\tilde{\mu}_{R}^{+} \tilde{\mu}_{R}^{-} \\
\tilde{\tau}_{1}^{+} \tilde{\tau}_{1}^{-} \\
\tilde{\nu}_{i}^{*} \tilde{\nu}_{i} \\
\chi_{1}^{0}\left(\chi_{1}^{0}, \chi_{2}^{0}\right) \\
\chi_{2}^{0} \chi_{2}^{0} \\
\chi_{1}^{+} \chi_{1}^{-} \\
\tau^{+} \tau^{-} \\
Z Z \\
Z W^{+} W^{-} \\
h h
\end{array}\right.
$$$$
A \rightarrow\left\{\begin{array} { l l } 
{ \tilde { \mu } _ { L } ^ { + } ( \tilde { \mu } _ { R } ^ { - } , \tilde { \tau } _ { 1 } ^ { - } ) + \text { c.c. } } \\
{ \tilde { \tau } _ { 2 } ^ { + } ( \tilde { \mu } _ { R } ^ { - } , \tilde { \tau } _ { 1 } ^ { - } ) + \text { c.c. } } \\
{ \chi _ { 1 } ^ { 0 } ( \chi _ { 1 } ^ { 0 } , \chi _ { 2 } ^ { 0 } ) } \\
{ \chi _ { 2 } ^ { 0 } \chi _ { 2 } ^ { 0 } } \\
{ \chi _ { 1 } ^ { + } \chi _ { 1 } ^ { - } } \\
{ \tau ^ { + } \tau ^ { - } } \\
{ Z h }
\end{array} \quad H ^ { + } \rightarrow \left\{\begin{array}{l}
\tilde{e}_{L}^{+} \tilde{\nu}_{e} \\
\tilde{\mu}_{L}^{+}\left(\tilde{\nu}_{\mu}, \tilde{\nu}_{\tau}\right) \\
\tilde{\tau}_{2}^{+}\left(\tilde{\nu}_{\mu}, \tilde{\nu}_{\tau}\right) \\
\tilde{\mu}_{R}^{+} \tilde{\nu}_{\mu} \\
\tilde{\tau}_{1}^{+}\left(\tilde{\nu}_{\mu}, \tilde{\nu}_{\tau}\right) \\
\chi_{1}^{+}\left(\chi_{1}^{0}, \chi_{2}^{0}\right) \\
\left(\tau^{+}, \mu^{+}\right) \nu \\
W^{+} h
\end{array}\right.\right.
$$

The above decay chains are subject to the following rules: a given decay marked with a superscript $(1-3)$ is

(1) avoided, if a $\chi_{2}^{0}$ was produced prior in the decay chain branch (i.e., assuming $m_{\chi_{2}^{0}} \simeq$ $\left.m_{\chi_{1}^{ \pm}}\right)$

(2) considered, if no prior slepton is present in the decay chain branch or if any prior slepton is mostly left-handed $\left(2^{*}\right.$ : if the prior slepton is left-handed, this further restricts the decay into only right-handed sleptons);

(3) considered, if no prior sneutrino appears in the corresponding branch of the decay chain.

Thus, for each final state, all possible allowed decay chains (i.e. present in the aforementioned list of decays) were generated, taking as a starting point the considered primary production modes. For the case of supersymmetric $e^{+} e^{-}$cross sections, as well as SUSY 
branching fractions, we used the public code SPheno [121]. We developed dedicated routines for the case of SUSY $e^{-} e^{-}$and SM cross sections, which were implemented onto SPheno.

Open Access. This article is distributed under the terms of the Creative Commons Attribution License which permits any use, distribution and reproduction in any medium, provided the original author(s) and source are credited.

\section{References}

[1] G.L. Fogli, E. Lisi, A. Marrone, A. Palazzo and A.M. Rotunno, Evidence of $\theta_{13}>0$ from global neutrino data analysis, Phys. Rev. D 84 (2011) 053007 [arXiv:1106.6028] [INSPIRE].

[2] P. Minkowski, $\mu \rightarrow$ e $\gamma$ at a rate of one out of 1-billion muon decays?, Phys. Lett. B 67 (1977) 421 [inSPIRE].

[3] M. Gell-Mann, P. Ramond and R. Slansky, Complex spinors and unified theories, in Supergravity, P. van Nieuwenhuizen and D.Z. Freedman eds., North-Holland, Amsterdam Netherlands (1979), pg. 315 [Print-80-0576 (CERN)] [INSPIRE].

[4] T. Yanagida, Horizontal symmetry and masses of neutrinos, in Proceedings of the Workshop on the Unified Theory and the Baryon Number in the Universe, O. Sawada and A. Sugamoto eds., KEK, Tsukuba Japan (1979), pg. 95 [INSPIRE].

[5] S.L. Glashow, The future of elementary particle physics, in Quarks and leptons, M. Lévy et al. eds., Plenum Press, New York U.S.A. (1980), pg. 687 [InSPIRE].

[6] R.N. Mohapatra and G. Senjanović, Neutrino mass and spontaneous parity violation, Phys. Rev. Lett. 44 (1980) 912 [INSPIRE].

[7] S. Davidson, E. Nardi and Y. Nir, Leptogenesis, Phys. Rept. 466 (2008) 105 [arXiv: 0802.2962] [INSPIRE].

[8] A. Abada, C. Biggio, F. Bonnet, M.B. Gavela and T. Hambye, Low energy effects of neutrino masses, JHEP 12 (2007) 061 [arXiv:0707.4058] [INSPIRE].

[9] R. Barbieri, D.V. Nanopoulos, G. Morchio and F. Strocchi, Neutrino masses in grand unified theories, Phys. Lett. B 90 (1980) 91 [inSPIRE].

[10] R.E. Marshak and R.N. Mohapatra, Selection rules for baryon number nonconservation in gauge models, talk given at Orbis Scientiae, Coral Gables U.S.A., 14-17 Jan 1980, VPI-HEP-80/02 [INSPIRE].

[11] T.P. Cheng and L.-F. Li, Neutrino masses, mixings and oscillations in $\mathrm{SU}(2) \times \mathrm{U}(1)$ models of electroweak interactions, Phys. Rev. D 22 (1980) 2860 [INSPIRE].

[12] M. Magg and C. Wetterich, Neutrino mass problem and gauge hierarchy, Phys. Lett. B 94 (1980) 61 [INSPIRE].

[13] G. Lazarides, Q. Shafi and C. Wetterich, Proton lifetime and fermion masses in an $\mathrm{SO}(10)$ model, Nucl. Phys. B 181 (1981) 287 [INSPIRE].

[14] J. Schechter and J.W.F. Valle, Neutrino masses in $\mathrm{SU}(2) \times \mathrm{U}(1)$ theories, Phys. Rev. D 22 (1980) 2227 [INSPIRE]. 
[15] R.N. Mohapatra and G. Senjanović, Neutrino masses and mixings in gauge models with spontaneous parity violation, Phys. Rev. D 23 (1981) 165 [InSPIRE].

[16] F. Borzumati and A. Masiero, Large muon and electron number violations in supergravity theories, Phys. Rev. Lett. 57 (1986) 961 [INSPIRE].

[17] J. Hisano, T. Moroi, K. Tobe and M. Yamaguchi, Lepton flavor violation via right-handed neutrino Yukawa couplings in supersymmetric standard model, Phys. Rev. D 53 (1996) 2442 [hep-ph/9510309] [INSPIRE].

[18] J. Hisano, T. Moroi, K. Tobe, M. Yamaguchi and T. Yanagida, Lepton flavor violation in the supersymmetric standard model with seesaw induced neutrino masses, Phys. Lett. B 357 (1995) 579 [hep-ph/9501407] [INSPIRE].

[19] J. Hisano and D. Nomura, Solar and atmospheric neutrino oscillations and lepton flavor violation in supersymmetric models with the right-handed neutrinos, Phys. Rev. D 59 (1999) 116005 [hep-ph/9810479] [INSPIRE].

[20] W. Buchmüller, D. Delepine and F. Vissani, Neutrino mixing and the pattern of supersymmetry breaking, Phys. Lett. B 459 (1999) 171 [hep-ph/9904219] [InSPIRE].

[21] Y. Kuno and Y. Okada, Muon decay and physics beyond the standard model, Rev. Mod. Phys. 73 (2001) 151 [hep-ph/9909265] [INSPIRE].

[22] J.R. Ellis, M.E. Gomez, G.K. Leontaris, S. Lola and D.V. Nanopoulos, Charged lepton flavor violation in the light of the Super-Kamiokande data, Eur. Phys. J. C 14 (2000) 319 [hep-ph/9911459] [INSPIRE].

[23] J.A. Casas and A. Ibarra, Oscillating neutrinos and $\mu \rightarrow e, \gamma$, Nucl. Phys. B 618 (2001) 171 [hep-ph/0103065] [INSPIRE].

[24] S. Lavignac, I. Masina and C.A. Savoy, $\tau \rightarrow \mu \gamma$ and $\mu \rightarrow e \gamma$ as probes of neutrino mass models, Phys. Lett. B 520 (2001) 269 [hep-ph/0106245] [INSPIRE].

[25] X.-J. Bi and Y.-B. Dai, Lepton flavor violation in the supersymmetric grand unified models with a lopsided mass matrix, Phys. Rev. D 66 (2002) 076006 [hep-ph/0112077] [INSPIRE].

[26] J.R. Ellis, J. Hisano, M. Raidal and Y. Shimizu, A new parametrization of the seesaw mechanism and applications in supersymmetric models, Phys. Rev. D 66 (2002) 115013 [hep-ph/0206110] [INSPIRE].

[27] F. Deppisch, H. Pas, A. Redelbach, R. Ruckl and Y. Shimizu, Probing the Majorana mass scale of right-handed neutrinos in mSUGRA, Eur. Phys. J. C 28 (2003) 365 [hep-ph/0206122] [INSPIRE].

[28] T. Fukuyama, T. Kikuchi and N. Okada, Lepton flavor violating processes and muon $g-2$ in minimal supersymmetric SO(10) model, Phys. Rev. D 68 (2003) 033012 [hep-ph/0304190] [INSPIRE].

[29] A. Brignole and A. Rossi, Anatomy and phenomenology of $\mu-\tau$ lepton flavor violation in the MSSM, Nucl. Phys. B 701 (2004) 3 [hep-ph/0404211] [INSPIRE].

[30] A. Masiero, S.K. Vempati and O. Vives, Massive neutrinos and flavor violation, New J. Phys. 6 (2004) 202 [hep-ph/0407325] [InSPIRE].

[31] T. Fukuyama, A. Ilakovac and T. Kikuchi, Lepton flavor violating leptonic/semileptonic decays of charged leptons in the minimal supersymmetric standard model, Eur. Phys. J. C 56 (2008) 125 [hep-ph/0506295] [INSPIRE]. 
[32] S.T. Petcov, W. Rodejohann, T. Shindou and Y. Takanishi, The see-saw mechanism, neutrino Yukawa couplings, LFV decays $l_{i} \rightarrow l_{j}+\gamma$ and leptogenesis,

Nucl. Phys. B 739 (2006) 208 [hep-ph/0510404] [INSPIRE].

[33] E. Arganda and M.J. Herrero, Testing supersymmetry with lepton flavor violating $\tau$ and $\mu$ decays, Phys. Rev. D 73 (2006) 055003 [hep-ph/0510405] [INSPIRE].

[34] F. Deppisch, H. Pas, A. Redelbach and R. Ruckl, Constraints on SUSY seesaw parameters from leptogenesis and lepton flavor violation, Phys. Rev. D 73 (2006) 033004 [hep-ph/0511062] [INSPIRE].

[35] C.E. Yaguna, Constraining mSUGRA parameters with $\mu \rightarrow e \gamma$ and $\mu$-e conversion in nuclei, Int. J. Mod. Phys. A 21 (2006) 1283 [hep-ph/0502014] [INSPIRE].

[36] L. Calibbi, A. Faccia, A. Masiero and S.K. Vempati, Lepton flavour violation from SUSY-GUTs: where do we stand for MEG, PRISM/PRIME and a super flavour factory, Phys. Rev. D 74 (2006) 116002 [hep-ph/0605139] [INSPIRE].

[37] S. Antusch, E. Arganda, M.J. Herrero and A.M. Teixeira, Impact of $\theta_{13}$ on lepton flavour violating processes within SUSY seesaw, JHEP 11 (2006) 090 [hep-ph/0607263] [INSPIRE].

[38] E. Arganda, M.J. Herrero and A.M. Teixeira, $\mu-e$ conversion in nuclei within the CMSSM seesaw: universality versus non-universality, JHEP 10 (2007) 104 [arXiv:0707.2955] [INSPIRE].

[39] E. Arganda, M.J. Herrero and J. Portoles, Lepton flavour violating semileptonic $\tau$ decays in constrained MSSM-seesaw scenarios, JHEP 06 (2008) 079 [arXiv:0803.2039] [INSPIRE].

[40] N. Arkani-Hamed, H.-C. Cheng, J.L. Feng and L.J. Hall, Probing lepton flavor violation at future colliders, Phys. Rev. Lett. 77 (1996) 1937 [hep-ph/9603431] [INSPIRE].

[41] I. Hinchliffe and F.E. Paige, Lepton flavor violation at the CERN LHC, Phys. Rev. D 63 (2001) 115006 [hep-ph/0010086] [INSPIRE].

[42] D.F. Carvalho, J.R. Ellis, M.E. Gomez, S. Lola and J.C. Romao, $\tau$ flavor violation in sparticle decays at the LHC, Phys. Lett. B 618 (2005) 162 [hep-ph/0206148] [INSPIRE].

[43] M.R. Buckley and H. Murayama, How can we test seesaw experimentally?, Phys. Rev. Lett. 97 (2006) 231801 [hep-ph/0606088] [INSPIRE].

[44] M. Hirsch, J.W.F. Valle, W. Porod, J.C. Romao and A. Villanova del Moral, Probing minimal supergravity in type-I seesaw with lepton flavour violation at the LHC, Phys. Rev. D 78 (2008) 013006 [arXiv:0804.4072] [INSPIRE].

[45] E. Carquin, J. Ellis, M.E. Gomez, S. Lola and J. Rodriguez-Quintero, Search for $\tau$ flavour violation at the LHC, JHEP 05 (2009) 026 [arXiv: 0812.4243] [INSPIRE].

[46] A.J. Buras, L. Calibbi and P. Paradisi, Slepton mass-splittings as a signal of LFV at the LHC, JHEP 06 (2010) 042 [arXiv:0912.1309] [INSPIRE].

[47] A. Abada, A.J.R. Figueiredo, J.C. Romao and A.M. Teixeira, Interplay of LFV and slepton mass splittings at the LHC as a probe of the SUSY seesaw, JHEP 10 (2010) 104 [arXiv: 1007.4833] [INSPIRE].

[48] A. Abada, A.J.R. Figueiredo, J.C. Romao and A.M. Teixeira, Probing the supersymmetric type-III seesaw: LFV at low-energies and at the LHC, JHEP 08 (2011) 099 [arXiv: 1104.3962] [INSPIRE]. 
[49] L. Calibbi, R.N. Hodgkinson, J. Jones Perez, A. Masiero and O. Vives, Flavour and collider interplay for SUSY at LHC7, Eur. Phys. J. C 72 (2012) 1863 [arXiv:1111.0176] [INSPIRE].

[50] L. Calibbi, R.N. Hodgkinson, J. Jones-Perez, A. Masiero and O. Vives, SUSY flavour at LHC7, PoS (EPS-HEP2011) 160 [arXiv:1111.6376] [INSPIRE].

[51] I. Galon and Y. Shadmi, Kinematic edges with flavor splitting and mixing, Phys. Rev. D 85 (2012) 015010 [arXiv:1108.2220] [INSPIRE].

[52] C. Arbelaez, M. Hirsch and L. Reichert, Supersymmetric mass spectra and the seesaw type-I scale, JHEP 02 (2012) 112 [arXiv:1112.4771] [INSPIRE].

[53] M.J. Herrero, J. Portoles and A.M. Rodriguez-Sanchez, Sensitivity to the Higgs sector of $S U S Y$-seesaw models in the lepton flavour violating $\tau \rightarrow \mu f_{0}(980)$ decay, Phys. Rev. D 80 (2009) 015023 [arXiv:0903.5151] [INSPIRE].

[54] ILC collaboration, J. Brau et al., ILC reference design report: ILC global design effort and world wide study, arXiv:0712.1950 [INSPIRE].

[55] ILC collaboration, G. Aarons et al., International Linear Collider reference design report volume 2: physics at the ILC, arXiv:0709.1893 [INSPIRE].

[56] ILD Concept Group-Linear Collider collaboration, T. Abe et al., The International Large Detector: letter of intent, arXiv:1006.3396 [INSPIRE].

[57] CLIC conceptual design report. Volume 2: Physics and detectors at CLIC, https://edms.cern.ch/file/1160419/2/CLIC_CDR_Review_080911.pdf.

[58] R.B. Palmer and R. Fernow, An overview of muon colliders, ICFA Beam Dyn. Newslett. 55 (2011) 22 [InSPIRE].

[59] J.-J. Blaising et al., Physics performances for scalar electrons, scalar muons and scalar neutrinos searches at CLIC, arXiv:1201.2092 [INSPIRE].

[60] H. Baer, C. Balázs, S. Hesselbach, J.K. Mizukoshi and X. Tata, Probing slepton mass nonuniversality at $e^{+} e^{-}$linear colliders, Phys. Rev. D 63 (2001) 095008 [hep-ph/0012205] [INSPIRE].

[61] A. Freitas, A. von Manteuffel and P.M. Zerwas, Slepton production at $e^{+} e^{-}$and $e^{-} e^{-}$ linear colliders, Eur. Phys. J. C 34 (2004) 487 [hep-ph/0310182] [InSPIRE].

[62] A. Freitas, A. von Manteuffel and P.M. Zerwas, Slepton production at $e^{+} e^{-}$and $e^{-} e^{-}$ linear colliders: addendum, Eur. Phys. J. C 40 (2005) 435 [hep-ph/0408341] [INSPIRE].

[63] A. Freitas, Feasibility of slepton precision measurements at a muon collider, arXiv:1107.3853 [INSPIRE].

[64] G. Moortgat-Pick, Impact of polarized $e^{-}$and $e^{+}$beams at a future linear collider and a Z-factory. Part I: fundamentals in polarization and electroweak precision physics, DESY-10-242 [INSPIRE].

[65] G. Moortgat-Pick, Impact of polarized $e^{-}$and $e^{+}$beams at a future linear collider and a Z-factory. Part II: physics beyond the standard model, DESY-10-243, [J. Phys. Conf. Ser. 298 (2011) 012001] [INSPIRE].

[66] LHC/LC Study Group collaboration, G. Weiglein et al., Physics interplay of the LHC and the ILC, Phys. Rept. 426 (2006) 47 [hep-ph/0410364] [INSPIRE]. 
[67] J.A. Aguilar-Saavedra, CP violation in $\tilde{\chi}_{1}^{0} \tilde{\chi}_{2}^{0}$ production at a linear collider, Nucl. Phys. B 697 (2004) 207 [hep-ph/0404104] [INSPIRE].

[68] J.A. Aguilar-Saavedra, Sneutrino cascade decays $\tilde{\nu}_{e} \rightarrow e^{-} \tilde{\chi}_{1}^{+} \rightarrow e^{-} f \bar{f}^{\prime} \tilde{\chi}_{1}^{0}$ as a probe of chargino spin properties and CP-violation, Nucl. Phys. B 717 (2005) 119 [hep-ph/0410068] [INSPIRE].

[69] O. Kittel, G. Moortgat-Pick, K. Rolbiecki, P. Schade and M. Terwort, Measurement of CP asymmetries in neutralino production at the ILC, Eur. Phys. J. C 72 (2012) 1854 [arXiv:1108.3220] [INSPIRE].

[70] J.A. Aguilar-Saavedra and A.M. Teixeira, Testing the Majorana nature of neutralinos in supersymmetric theories, Nucl. Phys. B 675 (2003) 70 [hep-ph/0307001] [INSPIRE].

[71] S.Y. Choi, M. Drees, A. Freitas and P.M. Zerwas, Testing the Majorana nature of gluinos and neutralinos, Phys. Rev. D 78 (2008) 095007 [arXiv: 0808.2410] [INSPIRE].

[72] M. Cannoni, S. Kolb and O. Panella, On the heavy Majorana neutrino and light sneutrino contribution to $e^{-} e^{-} \rightarrow \ell^{-} \ell^{-},(\ell=\mu, \tau)$, Eur. Phys. J. C 28 (2003) 375 [hep-ph/0209120] [INSPIRE].

[73] N. Arkani-Hamed, J.L. Feng, L.J. Hall and H.-C. Cheng, CP violation from slepton oscillations at the LHC and NLC, Nucl. Phys. B 505 (1997) 3 [hep-ph/9704205] [INSPIRE].

[74] J. Hisano, M.M. Nojiri, Y. Shimizu and M. Tanaka, Lepton flavor violation in the left-handed slepton production at future lepton colliders, Phys. Rev. D 60 (1999) 055008 [hep-ph/9808410] [INSPIRE].

[75] M. Guchait, J. Kalinowski and P. Roy, Supersymmetric lepton flavor violation in a linear collider: the role of charginos, Eur. Phys. J. C 21 (2001) 163 [hep-ph/0103161] [InSPIRE].

[76] M. Cannoni, S. Kolb and O. Panella, Lepton flavor violation in $e^{ \pm} e^{-} \rightarrow l^{ \pm} e^{-}(l=\mu, \tau)$ induced by $R$ conserving supersymmetry, Phys. Rev. D 68 (2003) 096002 [hep-ph/0306170] [INSPIRE].

[77] E. Carquin, J. Ellis, M.E. Gomez and S. Lola, Searches for lepton flavour violation at a linear collider, JHEP 11 (2011) 050 [arXiv:1106.4903] [INSPIRE].

[78] F. Deppisch, H. Pas, A. Redelbach, R. Ruckl and Y. Shimizu, The SUSY seesaw model and lepton flavor violation at a future electron positron linear collider, Phys. Rev. D 69 (2004) 054014 [hep-ph/0310053] [INSPIRE].

[79] F. Deppisch, J. Kalinowski, H. Pas, A. Redelbach and R. Ruckl, Supersymmetric lepton flavor violation at the $L H C$ and $L C$, hep-ph/0401243 [INSPIRE].

[80] I.F. Ginzburg, G.L. Kotkin, V.G. Serbo and V.I. Telnov, Colliding $\gamma e$ and $\gamma \gamma$ beams based on the single-pass $e^{ \pm} e^{-}$colliders (VLEPP type), Nucl. Instrum. Meth. 205 (1983) 47 [INSPIRE].

[81] I.F. Ginzburg, G.L. Kotkin, S.L. Panfil, V.G. Serbo and V.I. Telnov, Colliding $\gamma e$ and $\gamma \gamma$ beams based on single pass $e^{+} e^{-}$accelerators II. Polarization effects, monochromatization improvement, Nucl. Instrum. Meth. A 219 (1984) 5 [inSPIRE].

[82] M. Cannoni, C. Carimalo, W. Da Silva and O. Panella, Testing SUSY models of lepton flavor violation at a photon collider, Phys. Rev. D 72 (2005) 115004

[Erratum ibid. D 72 (2005) 119907] [hep-ph/0508256] [INSPIRE]. 
[83] T. Schwetz, M. Tortola and J.W.F. Valle, Where we are on $\theta_{13}$ : addendum to 'Global neutrino data and recent reactor fluxes: status of three-flavour oscillation parameters', New J. Phys. 13 (2011) 109401 [arXiv:1108.1376] [INSPIRE].

[84] DOUBLE-CHOOZ collaboration, Y. Abe et al., Indication for the disappearance of reactor electron antineutrinos in the Double CHOOZ experiment,

Phys. Rev. Lett. 108 (2012) 131801 [arXiv:1112.6353] [INSPIRE].

[85] T2K collaboration, M. Hartz, First oscillation results for the T2K experiment, arXiv: 1201.1846 [INSPIRE].

[86] MINOS collaboration, P. Adamson et al., An improved measurement of muon antineutrino disappearance in MINOS, Phys. Rev. Lett. 108 (2012) 191801 [arXiv:1202.2772] [INSPIRE].

[87] DAYA-BAY collaboration, F.P. An et al., Observation of electron-antineutrino disappearance at Daya Bay, Phys. Rev. Lett. 108 (2012) 171803 [arXiv:1203.1669] [INSPIRE].

[88] RENO collaboration, J.K. Ahn et al., Observation of reactor electron antineutrino disappearance in the RENO experiment, Phys. Rev. Lett. 108 (2012) 191802 [arXiv:1204.0626] [INSPIRE].

[89] ATLAS collaboration, G. Aad et al., Search for diphoton events with large missing transverse energy with $36 \mathrm{pb}^{-1}$ of $7 \mathrm{TeV}$ proton-proton collision data with the ATLAS detector, Eur. Phys. J. C 71 (2011) 1744 [arXiv:1107.0561] [InSPIRE].

[90] ATLAS collaboration, G. Aad et al., Search for supersymmetric particles in events with lepton pairs and large missing transverse momentum in $\sqrt{s}=7$ TeV proton-proton collisions with the ATLAS experiment, Eur. Phys. J. C 71 (2011) 1682 [arXiv:1103.6214] [INSPIRE].

[91] ATLAS collaboration, G. Aad et al., Search for an excess of events with an identical flavour lepton pair and significant missing transverse momentum in $\sqrt{s}=7 \mathrm{TeV}$ proton-proton collisions with the ATLAS detector, Eur. Phys. J. C 71 (2011) 1647 [arXiv: 1103.6208] [INSPIRE].

[92] ATLAS collaboration, G. Aad et al., Search for supersymmetry in pp collisions at $\sqrt{s}=7 \mathrm{TeV}$ in final states with missing transverse momentum and b-jets, Phys. Lett. B 701 (2011) 398 [arXiv:1103.4344] [INSPIRE].

[93] ATLAS collaboration, G. Aad et al., Search for stable hadronising squarks and gluinos with the ATLAS experiment at the LHC, Phys. Lett. B 701 (2011) 1 [arXiv:1103.1984] [INSPIRE].

[94] ATLAS collaboration, G. Aad et al., Search for squarks and gluinos using final states with jets and missing transverse momentum with the ATLAS detector in $\sqrt{s}=7 \mathrm{TeV}$ proton-proton collisions, Phys. Lett. B 701 (2011) 186 [arXiv:1102.5290] [INSPIRE].

[95] ATLAS collaboration, G. Aad et al., Search for supersymmetry using final states with one lepton, jets and missing transverse momentum with the ATLAS detector in $\sqrt{s}=7 \mathrm{TeV} p p$, Phys. Rev. Lett. 106 (2011) 131802 [arXiv:1102.2357] [INSPIRE].

[96] CMS collaboration, S. Chatrchyan et al., Search for supersymmetry at the LHC in events with jets and missing transverse energy, CERN-PH-EP-2011-138 (2011). 
[97] CMS collaboration, S. Chatrchyan et al., Search for supersymmetry in pp collisions at $\sqrt{s}=7 \mathrm{TeV}$ in events with a single lepton, jets and missing transverse momentum, JHEP 08 (2011) 156 [arXiv:1107.1870] [INSPIRE].

[98] CMS collaboration, S. Chatrchyan et al., Inclusive search for squarks and gluinos in pp collisions at $\sqrt{s}=7$ TeV, Phys. Rev. D 85 (2012) 012004 [arXiv:1107.1279] [InSPIRE].

[99] CMS collaboration, S. Chatrchyan et al., Search for new physics with jets and missing transverse momentum in pp collisions at $\sqrt{s}=7$ TeV, JHEP 08 (2011) 155 [arXiv:1106.4503] [INSPIRE].

[100] CMS collaboration, S. Chatrchyan et al., Search for supersymmetry in events with $b$ jets and missing transverse momentum at the LHC, JHEP 07 (2011) 113 [arXiv:1106.3272] [INSPIRE].

[101] CMS collaboration, S. Chatrchyan et al., Search for physics beyond the standard model using multilepton signatures in pp collisions at $\sqrt{s}=7$ TeV, Phys. Lett. B 704 (2011) 411 [arXiv:1106.0933] [INSPIRE].

[102] CMS collaboration, S. Chatrchyan et al., Search for supersymmetry in events with a lepton, a photon and large missing transverse energy in pp collisions at $\sqrt{s}=7 \mathrm{TeV}$, JHEP 06 (2011) 093 [arXiv:1105.3152] [INSPIRE].

[103] CMS collaboration, S. Chatrchyan et al., Search for new physics with same-sign isolated dilepton events with jets and missing transverse energy at the LHC, JHEP 06 (2011) 077 [arXiv:1104.3168] [INSPIRE].

[104] CMS collaboration, S. Chatrchyan et al., Search for physics beyond the standard model in opposite-sign dilepton events at $\sqrt{s}=7 \mathrm{TeV}$, JHEP 06 (2011) 026 [arXiv:1103.1348] [INSPIRE].

[105] CMS collaboration, S. Chatrchyan et al., Search for supersymmetry in pp collisions at $\sqrt{s}=7 \mathrm{TeV}$ in events with two photons and missing transverse energy, Phys. Rev. Lett. 106 (2011) 211802 [arXiv:1103.0953] [InSPIRE].

[106] CMS collaboration, V. Khachatryan et al., Search for supersymmetry in pp collisions at $7 \mathrm{Te} V$ in events with jets and missing transverse energy, Phys. Lett. B 698 (2011) 196 [arXiv:1101.1628] [INSPIRE].

[107] CMS collaboration, V. Khachatryan et al., Search for stopped gluinos in pp collisions at $\sqrt{s}=7$ TeV, Phys. Rev. Lett. 106 (2011) 011801 [arXiv:1011.5861] [INSPIRE].

[108] Particle Data Group collaboration, C. Amsler et al., Review of particle physics, Phys. Lett. B 667 (2008) 1 [INSPIRE].

[109] MEG collaboration, J. Adam et al., New limit on the lepton-flavour violating decay $\mu^{+} \rightarrow e^{+} \gamma$, Phys. Rev. Lett. 107 (2011) 171801 [arXiv:1107.5547] [INSPIRE].

[110] Mu2E collaboration, D. Glenzinski, The Mu2e experiment at Fermilab, AIP Conf. Proc. 1222 (2010) 383 [inSPIRE].

[111] COMET collaboration, Y.G. Cui et al., Conceptual design report for experimental search for lepton flavor violating $\mu^{-}-e^{-}$conversion at sensitivity of $10^{-16}$ with a slow-extracted bunched proton beam (COMET), KEK-2009-10 [INSPIRE].

[112] B. Pontecorvo, Mesonium and anti-mesonium, Sov. Phys. JETP 6 (1957) 429 [Zh. Eksp. Teor. Fiz. 33 (1957) 549] [INSPIRE]. 
[113] Z. Maki, M. Nakagawa and S. Sakata, Remarks on the unified model of elementary particles, Prog. Theor. Phys. 28 (1962) 870 [InSPIRE].

[114] LHCB collaboration, R. Aaij et al., Strong constraints on the rare decays $B_{s} \rightarrow \mu^{+} \mu^{-}$and $B^{0} \rightarrow \mu^{+} \mu^{-}$, Phys. Rev. Lett. 108 (2012) 231801 [arXiv:1203.4493] [INSPIRE].

[115] D. Larson et al., Seven-year Wilkinson Microwave Anisotropy Probe (WMAP) observations: power spectra and WMAP-derived parameters, Astrophys. J. Suppl. 192 (2011) 16 [arXiv: 1001.4635] [INSPIRE].

[116] M. Raidal et al., Flavour physics of leptons and dipole moments, Eur. Phys. J. C 57 (2008) 13 [arXiv:0801.1826] [INSPIRE].

[117] M.C. Gonzalez-Garcia, M. Maltoni and J. Salvado, Updated global fit to three neutrino mixing: status of the hints of $\theta_{13}>0$, JHEP 04 (2010) 056 [arXiv: 1001.4524] [INSPIRE].

[118] H. Baer and J. List, Post LHC7 SUSY benchmark points for ILC physics, arXiv: 1205.6929 [INSPIRE].

[119] S.S. AbdusSalam et al., Benchmark models, planes, lines and points for future SUSY searches at the LHC, Eur. Phys. J. C 71 (2011) 1835 [arXiv:1109.3859] [InSPIRE].

[120] G. Bélanger, F. Boudjema, A. Pukhov and A. Semenov, Dark matter direct detection rate in a generic model with MicrOMEGAs 2.2, Comput. Phys. Commun. 180 (2009) 747 [arXiv:0803.2360] [INSPIRE].

[121] W. Porod, SPheno, a program for calculating supersymmetric spectra, SUSY particle decays and SUSY particle production at $e^{+} e^{-}$colliders, Comput. Phys. Commun. 153 (2003) 275 [hep-ph/0301101] [INSPIRE].

[122] ATLAS collaboration, G. Aad et al., Combined search for the standard model Higgs boson using up to $4.9 \mathrm{fb}^{-1}$ of pp collision data at $\sqrt{\mathrm{s}}=7 \mathrm{TeV}$ with the ATLAS detector at the LHC, Phys. Lett. B 710 (2012) 49 [arXiv: 1202.1408] [INSPIRE].

[123] CMS collaboration, S. Chatrchyan et al., Combined results of searches for the standard model Higgs boson in pp collisions at $\sqrt{s}=7 \mathrm{TeV}$, Phys. Lett. B 710 (2012) 26 [arXiv: 1202.1488] [INSPIRE].

[124] A. Arbey, M. Battaglia, A. Djouadi, F. Mahmoudi and J. Quevillon, Implications of a 125 GeV Higgs for supersymmetric models, Phys. Lett. B 708 (2012) 162 [arXiv: 1112.3028] [INSPIRE].

[125] M. Battaglia et al., Physics performances for scalar electrons, scalar muons and scalar neutrinos searches at CLIC, LCD-Note-2011-018 (2011). 